\title{
Statistical methods for the prospective detection of infectious disease outbreaks: A review
}

\author{
Steffen Unkel ${ }^{\dagger 1}$, C. Paddy Farrington ${ }^{1}$, \\ Paul H. Garthwaite ${ }^{1}$, Chris Robertson ${ }^{2}$ and Nick Andrews ${ }^{3}$ \\ ${ }^{1}$ Department of Mathematics and Statistics \\ The Open University, Milton Keynes, UK \\ ${ }^{2}$ Department of Mathematics and Statistics \\ University of Strathclyde, Glasgow, UK \\ ${ }^{3}$ Statistics Unit, Centre for Infections \\ Health Protection Agency, London, UK
}

August 19, 2010

\begin{abstract}
Unusual clusters of disease must be detected rapidly for effective public health interventions to be introduced. Over the past decade there has been a surge in interest in statistical methods for the early detection of infectious disease outbreaks. This growth in interest has given rise to much new methodological work, ranging across the spectrum of statistical methods. This paper presents a comprehensive review of the statistical approaches that have been proposed. Applications to both laboratory and syndromic surveillance data are provided to illustrate the various methods.
\end{abstract}

Keywords: Biosurveillance; Clusters; Control chart; Epidemics; Infectious diseases; Outbreak; Prospective detection; Surveillance.

\footnotetext{
${ }^{\dagger}$ Correspondence should be addressed to: Steffen Unkel, Department of Mathematics and Statistics, Faculty of Mathematics, Computing \& Technology, The Open University, Walton Hall, Milton Keynes, MK7 6AA, United Kingdom (e-mail:S.Unkel@open.ac.uk).
} 


\section{Setting the scene}

The past decade has witnessed a large increase in research activity on the statistical issues related to prospective detection of outbreaks of infectious diseases. The major challenges in this expanding field derive from its focus on prospective detection, namely, detection of outbreaks as they arise, in a sufficiently timely fashion to enable effective control measures to be taken. The growth in this area, sometimes now referred to as biosurveillance (e.g., Shmueli and Burkom 2010), has been so rapid as to spawn conferences and a learned society, the International Society for Disease Surveillance (http://www.syndromic.org), founded in 2005 .

Outbreak investigations go back at least to John Snow's iconic removal of the handle of London's Broad Street pump during the 1854 cholera epidemic. In the modern era, following a trend apparent in all areas of epidemiology, statistical methods have come to the fore in outbreak detection and control, though for a long time the main use of statistical techniques in outbreak epidemiology was in identifying their source. Nevertheless, for several decades (see Tillett and Spencer (1982) for an early example), statistical techniques have also been used to provide early warnings of outbreaks, supplementing more traditional surveillance based on a network of alert public health physicians. Since the early 1990s, the increasingly widespread availability of computerised databases which can be interrogated for evidence of emerging outbreaks has greatly facilitated the use of statistical outbreak detection, and has witnessed the creation of automated detection systems to process data on very large numbers of infections at frequent time intervals. Since the turn of the twenty-first century, two factors have combined to give further impetus to developments in this area: new concerns about the possible threat of large-scale bioterrorism, and heightened public and media awareness about emerging or re-emerging infections, including hospital-acquired infections such as methicillin-resistant Staphylococcus aureus (MRSA) and Clostridium difficile, and global epidemics such as severe acute respiratory syndrome (SARS) in 2002-2003 and the 2009 H1N1 swine influenza pandemic.

Under these influences, a new focus has emerged, namely the surveillance of syndromes, which complements the previous emphasis on surveillance of infections. Thus, much of the new literature in the field relates to syndromic surveillance, which exploits more diverse 
sources of data, such as calls to telephone or internet helplines, medical consultations, and pharmacy sales, that are believed to reflect in more timely fashion changes in behaviour that may stem from a large-scale bioterrorist incident. So far, no such incident has occurred. However, the need to detect outbreaks from more mundane (but frequently occurring) sources such as contaminated foodstuffs, breakdowns in water treatment plants, low vaccine efficacy, or imported infections - remains, and has led to further developments, as witnessed by the inclusion of routine methods for statistical outbreak detection in laboratory-based surveillance systems in several European countries (Hulth et al. 2010).

Both syndromic and laboratory-based prospective surveillance methods for outbreak detection pose diverse statistical challenges, relating to data sources, evaluation, multiplicity control, and follow-up, as well as the statistical techniques used to detect anomalies in data series. Periodic reviews of these methods, with varying emphases, have appeared in the statistical literature, notably Sonesson and Bock (2003), Farrington and Andrews (2004), Buckeridge et al. (2005) and Shmueli and Burkom (2010). There have also been numerous developments in related fields such as pharmacovigilance and institutional performance monitoring.

The aim of the present review is to provide an up-to-date, comprehensive account of the statistical methods that have been proposed for detecting anomalies in data series, specifically in the context of prospective outbreak detection. Our aim is not to cover the entire range of statistical issues relevant to outbreak detection (Fienberg and Shmueli 2005) - comprehensive coverage is probably no longer possible in a single review paper. Rather, we focus entirely on statistical methods for detecting aberrations. Our motivation for doing so is to inform a detailed study of some of the outbreak detection systems used in the UK. The paper is structured in the following Sections, defined purely for convenience: regression techniques (Section 2), time series methodology (Section 3), methods inspired by statistical process control (Section 4), methods incorporating spatial information (Section 5), and multivariate outbreak detection (Section 6). Concluding comments are given in Section 7. 


\section{Regression techniques}

Regression methods of outbreak detection are simple to apply and to automate. They have been widely used, both for detecting outbreaks in surveillance systems based on laboratory reports and notified infections, and for syndromic surveillance. Regression methods can be regarded as extending the Shewhart chart (Shewhart 1931), in which a process variable $y_{t}$ which is normally distributed $\mathcal{N}\left(\mu, \sigma^{2}\right)$ when in control is monitored by tracking the values of $y_{t}-\mu$, an alert being declared when $\left|y_{t}-\mu\right|>k \sigma$ for some pre-specified value of $k{ }^{1}$

When applied to outbreak detection, only the upper control limit $\mu+k \sigma$ is usually of interest. Regression methods generalize the Shewhart chart in three respects: the in-control mean $\mu$ and possibly the in-control standard deviation $\sigma$ vary with time; both these quantities must be estimated from historical data; and the distribution of $y_{t}$ may not be normal.

The performance of regression outbreak detection methods may be expected to reflect the performance of Shewhart charts: applied to an observation at time $t$, they are effective at detecting large outbreaks starting at time $t$, but rather less effective at detecting more gradual outbreaks starting at some time earlier than $t$.

In Subsection 2.1 and Subsection 2.2 parametric and semiparametric regression methods are described. Most regression methods are based on a threshold value, above which reports are declared aberrant. How these thresholds may be obtained is explained in Subsection 2.3, whereas in Subsection 2.4 non-thresholding methods are considered.

\subsection{Parametric models}

Perhaps the simplest regression model for outbreak detection is that described by Stroup et al. (1989), in which the expected disease count at time $t, \mathrm{E}\left(y_{t}\right)$, is calculated as the mean of observed counts at times $t-1, t, t+1$ over some pre-specified number of years. This ensures that seasonal effects are automatically adjusted for by design rather than by explicit modelling, thus providing some element of robustness. However, this model does not incorporate time trends. Stroup et al. (1989) apply this model, using normal errors, to data

\footnotetext{
${ }^{1}$ Throughout the paper, random variables and their realizations are not distinguished by using uppercase and lowercase letters. This is because in the multivariate case the reader will be more concerned about whether a vector or matrix of data is involved than with the distinction between random variables and their observed values.
} 
on notifiable infections. The results are summarized in a simple graphic published routinely in Morbidity and Mortality Weekly Report of the Centers for Disease Control and Prevention (CDC) (Stroup et al. 1993).

A commonly used fully parametric outbreak detection regression model is based on that of Serfling (1963), who modelled historical baselines using a trigonometric function with linear trend of the form:

$$
\mathrm{E}\left(y_{t}\right)=\mu+\alpha t+\sum_{i=1}^{r}\left\{\beta_{i} \sin \left(\omega_{i} t\right)+\gamma_{i} \cos \left(\omega_{i} t\right)\right\}
$$

and normal errors with constant variance. Serfling (1963) used the regression equation (1) to estimate excess mortality due to influenza based on weekly data on pneumoniainfluenza deaths. This model has subsequently been used to detect the onset of epidemics of influenza (Costagliola et al. 1991, 1994). An automated version of the Serfling model with cubic trend and three trigonometric terms (i.e. $r=3$ ), with model selection based on the Akaike information criterion (AIC), has been developed for prospective and retrospective surveillance, and is available as a web-based application (Pelat et al. 2007) at http://www.u707.jussieu.fr/periodic_regression. Figure 1 shows a sample output screen from the system of Pelat et al. (2007), displaying the result of a prospective analysis of weekly counts of Salmonella enteritidis Phage type (PT) 4 in England, Wales and Northern Ireland from 2000 to 2009 and model-based extrapolation for the year 2010 with an epidemic threshold. The user has first to select a subset of the whole data series (called 'training period') which is used to estimate the baseline level. In Figure 1 the training period consists of the years 2006 to 2009 . Then the $20 \%$ highest values in the training data are excluded to account for past outbreaks (default value: 15\%). A Serfling-type regression equation is used to model the baseline. A threshold is obtained by taking an upper percentile for the prediction distribution, here the upper 90th percentile is chosen. The system declares an outbreak as soon as an observation exceeds this threshold.

When data are sparse, the normal errors regression model is inappropriate. Parker (1989) instead used Poisson regression with logarithmic link to monitor the mortality associated with abortions. Such a model can be elaborated at will; Jackson et al. (2007) describe a Poisson log-linear model for syndromic surveillance with terms for day of the week, month, linear time trend, and holidays. If denominator population data are available, binomial lo- 


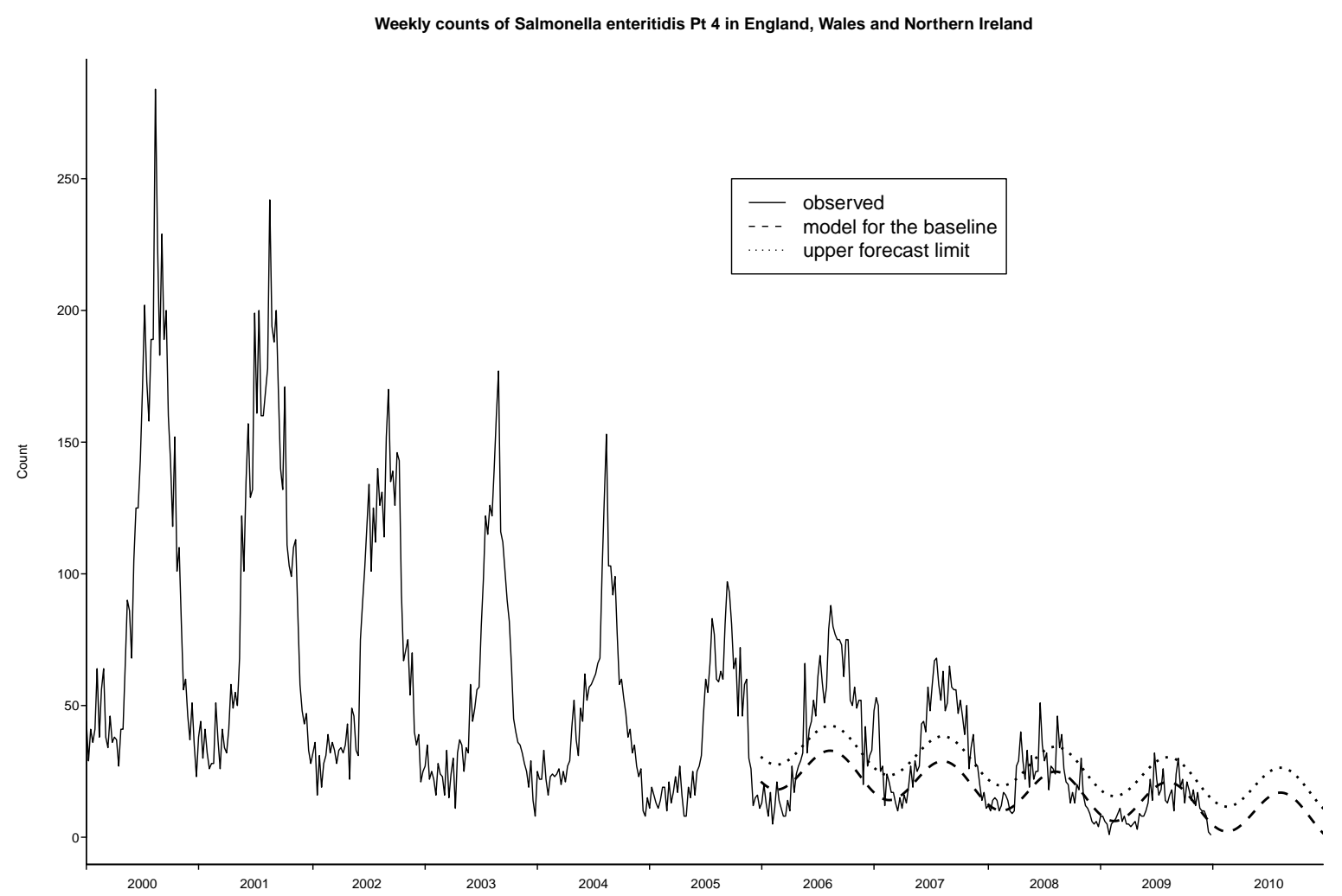

Figure 1: Sample output screen from the system of Pelat et al. (2007) (see text for explanation).

gistic models can be fitted in much the same way, the flexibility of the generalised linear model (GLM) approach allowing further extensions to include random effects, for example to represent spatial variation (cf. Section 5).

The log-linear regression model of Farrington et al. (1996), like that of Stroup et al. (1989), adjusts for seasonal effects by design, and explicitly allows for linear trends. Since much surveillance data exhibiting considerable overdispersion, the model is quasi-Poisson with

$$
\mathrm{E}\left(y_{t}\right)=\mu_{t}, \quad \log \left(\mu_{t}\right)=\alpha+\beta t, \quad \operatorname{var}\left(y_{t}\right)=\phi \mu_{t},
$$

where $\phi$ is the dispersion parameter. To try to reduce the influence of past outbreaks, baseline values with high residuals are given lower weights in the regression. Estimates of model parameters in (2) are obtained by a quasi-likelihood method.

The method of Farrington et al. (1996) is used routinely by the Health Protection Agency (HPA) to detect outbreaks in laboratory-based surveillance data in England and Wales, and 
is referred to as the Lab-Base exceedance system hereafter. Elaborations of the Lab-Base exceedance system have been described for use with laboratory data in Scotland (McCabe et al. 2003) and The Netherlands (Widdowson et al. 2003).

\subsection{Semiparametric models}

All the regression models so far described use a parametric model to represent the historical data. A contrasting strategy is to use a non-parametric model for the historical baseline, as widely used in monitoring mortality and other effects in environmental time series (Dominici et al. 2003). The SPOT (Salmonella Potential Outbreak Targeting) system described by Stern and Lightfoot (1999) uses a smoothing method to obtain baselines and standard deviation. Five years' historical data are first smoothed, and the baseline value for each time point in the yearly cycle is taken to be the median of the five smoothed values. The standard deviation is obtained by smoothing the residuals (raw values minus smooth values). This system implicitly assumes Gaussian errors by using an alarm threshold of two standard deviations above the baseline (with a filter for low counts).

Wieland et al. (2007) proposed to model both the mean $\mu_{t}$ and the variance $\sigma_{t}^{2}$ at each time point $t$, using separate generalized additive models for both quantities. First, a generalized additive model (GAM) is fitted to historical data to obtain $\hat{\mu}_{t}$. Then a second GAM is fitted to the residuals from the first GAM in order to obtain $\hat{\sigma}_{t}^{2}$. The threshold is taken as $\hat{\mu}_{t}+k \hat{\sigma}_{t}$ for some choice of $k$. The smooth terms in the GAMs were based on Gaussian kernel smoothers, with bandwidth chosen so as to minimize the mean predictive squared error on the historical data.

Mean-regression methods lack robustness to the presence of outbreaks in the baseline values, which bias $\hat{\mu}_{t}$ upwards and hence reduce the sensitivity of the system. Sensitivity is the probability that a true outbreak is detected: a high sensitivity is desirable for outbreaks of public health importance. Serfling (1963) identified past outbreaks by visual inspection and omitted them from the model; this will tend to bias $\hat{\mu}_{t}$ downwards and thus reduce the specificity. Specificity is the probability that a time period without an outbreak is correctly identified as such. The complement of this, 1 - specificity, must be kept low to avoid user fatigue and loss of credibility. The Lab-Base exceedance system (Farrington et al. 1996) 
downweights outliers in the baselines, but this reduces rather than eliminates the bias, as do non-parametric smoothing techniques.

An alternative is to use a wavelet transform of the baseline values to account for low frequency variation due to trends and seasonality, while remaining robust to high frequency variation resulting from past outbreaks or other artefacts, such as holiday dips. This approach was proposed by Zhang et al. (2003), whose simple and hence easily automated Wavelet-based Anomaly Detector (WAD) subtracts a baseline value obtained using the wavelet transform and bases thresholds on the distribution of the residuals (see also Wieland et al. 2007). More complex wavelet-based methods, which may also be applied in a time series framework, were used by Goldenberg et al. (2002) and discussed in Shmueli (2005).

\subsection{Obtaining the thresholds}

Most regression-based methods specify a model for the mean at time $t$, and declare an alarm at time $t$ if the observed value lies above some threshold determined by the mean and variance. A commonly used procedure is to estimate the baseline value $\hat{\mu}_{t}$ and the process variance at time $t, \hat{\sigma}_{t}^{2}$, and, assuming normal errors, to define the upper threshold by

$$
u_{t}=\hat{\mu}_{t}+k \hat{\sigma}_{t}
$$

where in many applications it is further assumed that the process variance is constant, $\sigma_{t}^{2}=$ $\sigma^{2}$. A more accurate approach is to base the threshold on an upper $100(1-\alpha) \%$ prediction limit which takes account both of the process variance and the uncertainty in the estimation of the baseline value. Thus, for example, the Poisson model of Parker (1989) combines the estimated process variance $\hat{\mu}_{t}$ with the regression error variance $\operatorname{var}(\hat{\mu})=\hat{\mu}^{2} \operatorname{var}\left(\log \left(\hat{\mu}_{t}\right)\right)$, obtained using the delta method, to obtain the total variance

$$
\operatorname{var}_{T}\left(y_{t}\right)=\hat{\mu}_{t}\left\{1+\hat{\mu}_{t} \operatorname{var}\left(\log \left(\hat{\mu}_{t}\right)\right)\right\}
$$

The quasi-Poisson method of Farrington et al. (1996) uses a similar approach. Clearly, regression methods do not account for serial correlation in the baselines. Kafadar and Stroup (1992) investigated bootstrap and jacknife procedures to estimate $\operatorname{var}\left(\hat{\mu}_{t}\right)$ but suggested that such adjustments are inadvisable unless the autocorrelation structure is known or can be well estimated. 
A further problem with thresholds of the form $\mu_{t}+k \sigma$ is that the normal theory upon which they are based is usually inappropriate, especially when the background means $\mu_{t}$ are small. The accuracy of the threshold - namely the extent to which $\mathrm{P}\left(y_{t}>u_{t}\right)$ matches the nominal $100 \alpha \%$ level upon it is based - will usually vary with $\mu_{t}$, hence the sensitivity and specificity will vary with $\mu_{t}$. This is undesirable for systems designed to monitor several different data series with a wide range of expected values.

Consequently, some detection algorithms apply a transformation to approximate normality, or approximate symmetry, derive the threshold on the transformed scale, and transform the threshold back to the original scale. The quasi-Poisson method of Farrington et al. (1996) uses the $2 / 3$ power transformation, which yields approximate symmetry for Poisson data. The upper threshold is defined as

$$
u_{t}=\hat{\mu}_{t}\left\{1+\frac{2}{3} z_{\alpha}\left(\frac{\hat{\phi}_{t} \hat{\mu}_{t}+\operatorname{var}\left(\hat{\mu}_{t}\right)}{\hat{\mu}_{t}^{2}}\right)^{1 / 2}\right\}^{3 / 2},
$$

where $z_{\alpha}$ is the $100(1-\alpha)$-percentile of the standard normal distribution. The rationale is that, for rare organisms, $\phi \simeq 1$ and counts are distributed approximately Poisson, while for frequent organisms normal approximations are valid. There are many possibilities: for example Cooper et al. (2004) use a sinh-based transformation applied to a binomial proportion to achieve a similar result.

\subsection{Non-thresholding methods}

Most regression methods for outbreak detection use a threshold $u_{t}$ at time $t$ to determine whether the current observation $y_{t}$ is aberrant. An alternative is to test the null hypothesis that $y_{t}$ belongs to the same distribution as the baseline values; Parker (1989) discusses various tests, including the likelihood ratio test, for Poisson data.

Other criteria for detecting outbreaks may be specified, based on the qualitative features of an outbreak, such as the start of an outbreak, at which point a previously stationary time series begins to increase, and the peak of an outbreak, at which the counts stop increasing and start decreasing (Andersson et al. 2008). Bock et al. (2008) describe methods for identifying the peak of an epidemic, and Frisén and Andersson (2009) and Frisén et al. (2010a, 2009) describe parametric and semi-parametric regression methods for detecting the onset of an 
epidemic.

The semiparametric model to detect epidemic onset assumes that the disease counts belong to a specified distribution within the regular exponential family. Under the semiparametric version of the model, it is assumed that the disease counts $y_{s}$ up to time $t(s \leq t)$ either have constant mean (the null hypothesis), or increase monotonically from time $t=d$ where $d$ is known (the alternative hypothesis). For Poisson counts that increase from the start of the series $(d=1)$, the likelihood ratio statistic is

$$
L R=\prod_{s=1}^{t}\left(\frac{\hat{\mu}_{s}^{1}}{\hat{\mu}_{s}^{0}}\right)^{y_{s}},
$$

where $\hat{\mu}_{s}^{0}$ is the maximum likelihood estimator (MLE) of the process mean at time $s$ under the null hypothesis, and $\hat{\mu}_{s}^{1}$ is the MLE under the alternative hypothesis. An alert is declared if $L R>k$ for some pre-specified value $k$. Frisén et al. (2010a, 2009) describe applications to outbreaks of influenza and tularaemia in Sweden. Computer software for this surveillance method is available (http://www.statistics.gu.se/surveillance), called 'Outbreak Detection P', as both a SAS program and as a VBA (Visual Basic for Applications) macro in Microsoft Excel for Windows. Figure 2 shows the 'alarm graph' obtained from an analysis of the weekly counts of Salmonella enteritidis PT 4 in England, Wales and Northern Ireland in the year 2009 by means of the 'Outbreak Detection P' program. The likelihood ratio (alarm) statistic (3) exceeds the warning limit (100) for the first time in week 24 and the alarm limit (5000) in week 25. The semiparametric method by Frisén and Andersson (2009) is also implemented in the R software package surveillance (Höhle 2007; Höhle and Mazick 2010). The surveillance package provides interfaces of several well-known procedures for prospective outbreak detection, among others the ones of Stroup et al. (1989), Farrington et al. (1996) and the system used by the Robert Koch Institute, Germany (Hulth et al. 2010). For a complete overview of its content, see http://surveillance.r-forge.r-project.org.

\section{Time series methodology}

Unlike most regression techniques, time series methods acknowledge the correlation structure of the data. For syndromic and laboratory data which are generally collected daily or weekly the principal correlations are autocorrelations at a lag of 1 time period (serial 


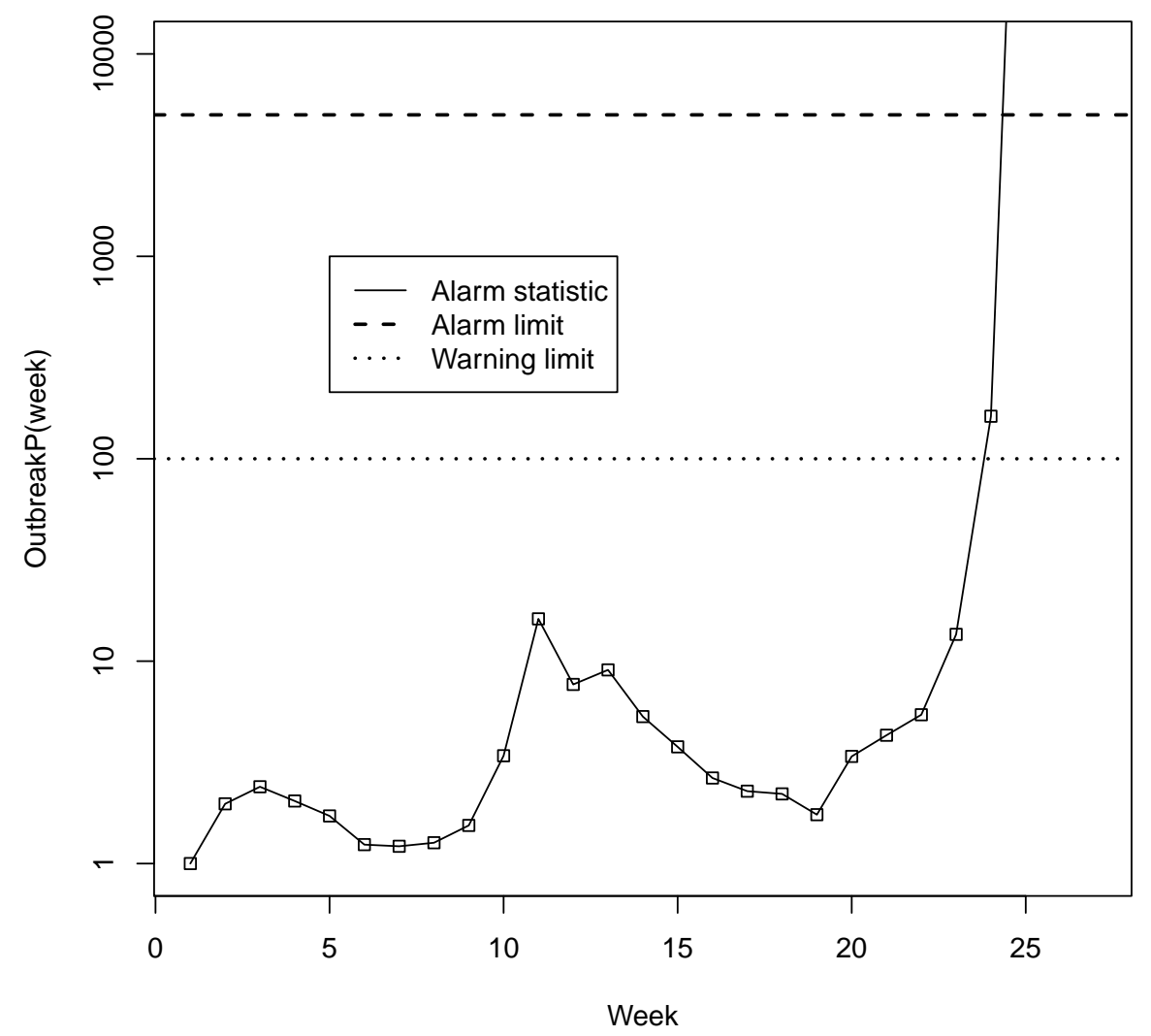

Figure 2: Sample alarm graph obtained from the 'Outbreak Detection P' program (see text for explanation).

correlation) and correlations associated with the seasonal pattern in the data, which can be a combination of weekly or yearly seasonality. When times series data are available over a relatively long period of time, it is important to estimate the trend and seasonal components as the autocorrelation structure can only be identified using a stationary time series. Methods for estimating the trend and seasonal effects are described in Subsection 3.1. Failure to properly account for the autocorrelation will result in a misspecified model which may have bias in the estimated effects and prediction intervals which are too narrow, leading to a larger number of potential exceptions. The Box-Jenkins type methodology was designed to take the autocorrelation structure into account. These models are considered in Subsection 3.2. Much statistical innovation in the field of outbreak detection has tended towards time series models of increasing complexity, including Bayesian and hidden Markov models. The latter are discussed in Subsection 3.3. 


\subsection{Trend and seasonal estimation}

With outbreak surveillance, the estimation of trend is best accomplished through a relatively simple procedure that is flexible and does not make any great demands on intervention by the operator. For time series, where there is a considerable amount of historical data and where the seasonal pattern is regular, a Serfling model (Serfling 1963) based upon sines and cosines may be used to estimate the trend and seasonal component.

Two common time series methods used in surveillance are simple exponential smoothing (e.g., Healy 1983; Ngo et al. 1996) and the Holt-Winters procedure (Holt 1957; Winters 1960). Simple exponential smoothing assures the data have no trend or seasonality. It forms predictions by taking a weighted average of past observations, where the weights decrease exponentially the further they are into the past (cf. Section 4).

The Holt-Winters technique is a generalization of simple exponential smoothing that allows for local trend and seasonal factors. Thus, for a series with multiplicative seasonal variation of period $p$ (e.g. for monthly observation $p=12$ ), the $h$-step prediction function for the Holt-Winters model is given by (Chatfield and Yar 1988):

$$
\hat{y}_{t+h}=\left(a_{t}+h b_{t}\right) s_{t-p+h} \quad(h=1, \ldots, p),
$$

where $a_{t}$ is the overall average level of the series, $b_{t}$ represents the trend and $s_{t}$ represents the seasonal component, given by

$$
\begin{aligned}
a_{t} & =\alpha \frac{y_{t}}{s_{t-p}}+(1-\alpha)\left(a_{t-1}+b_{t-1}\right), \\
b_{t} & =\beta\left(a_{t}-a_{t-1}\right)+(1-\beta) b_{t-1}, \\
s_{t} & =\gamma \frac{y_{t}}{a_{t}}+(1-\gamma) s_{t-p} .
\end{aligned}
$$

There are analogous formulae for additive seasonal variation. The smoothing constants $\alpha$, $\beta$ and $\gamma(0<\alpha, \beta, \gamma<1)$ in $(5)-(7)$ can be estimated by minimizing the sum of squares of the one-step ahead prediction errors.

Holt-Winters is an extremely flexible method for estimating trend and has been used in many surveillance systems. It has performed well in many forecasting competitions in comparison to other more complex methods (Chatfield and Yar 1988). In syndromic surveillance systems it has been used to model outpatient attendance, in comparison to adaptive (using a moving baseline window) and non-adaptive regression models (Burkom et al. 2007), and found to 
provide a better fit to the data. It has also been used to account for the effects of temporal autocorrelation and spatial correlation when modelling calls to NHS24 and laboratory reports (Wagner 2010).

\subsection{ARIMA and INAR models}

Autoregressive integrated moving average (ARIMA) models (Box and Jenkins 1970) have been used for detecting outbreaks of infectious disease (e.g., Choi 1981; Helfenstein 1986; Reis and Mandl 2003; Watier et al. 1991). Fitting an ARIMA model requires the time series to be stationary. As much syndromic and laboratory data are likely to have seasonal and trend components it is necessary to remove these components from the original data before estimating the autocorrelation. Furthermore, the statistical testing procedure is based upon the normal distribution and this will really only be valid for infections or syndromes which occur frequently. When using outbreak surveillance for relatively rare events, or for more common events in smaller areas, then integer-valued methods may be more appropriate.

The integer-valued autoregressive (INAR) model (e.g., Weiß 2009) is based upon the convolution operator o (Steutel and van Harn 1979), where

$$
\alpha \circ x=\sum_{k=1}^{x} y_{k},
$$

and the $y_{k}$ are independent and identically distributed Bernoulli random variables with probability $\mathrm{P}\left(y_{k}=1\right)=\alpha$ and $x$ is a non-negative discrete random variable. Using (8), an INAR model of order $p$, denoted by $\operatorname{INAR}(p)$, can be defined as

$$
x_{t}=\sum_{i=1}^{p} \alpha_{i} \circ x_{t-i}+\epsilon_{t},
$$

where $\epsilon_{t}$ is a (non-negative) random shock. It follows from (9) that an $\operatorname{INAR}(1)$ model is:

$$
\begin{aligned}
x_{t} & =\alpha_{1} \circ x_{t-1}+\epsilon_{t} \\
& =y_{1}+y_{2}+\ldots+y_{x_{t-1}}+\epsilon_{t} .
\end{aligned}
$$

The model (10) states that the number of new cases in the interval $(t-1, t]$ is made up of two components - the $x_{t-1}$ cases transmit the infection independently with probability $\alpha_{1}$ and, as a consequence, $\sum y_{k}$ new cases arise, and a random number $\epsilon_{t}$ of new cases are generated via independent sources. Parameter estimates may be obtained using, for example, 
the Yule-Walker estimation technique.

When fitting ARIMA and INAR models to meningococcal incidence in the Montreal region of Canada (Cardinal et al. 1999), INAR(5) and AR(5) models were required for data aggregated in thirteen 4 week periods each year, from 1986 to 1993. There was no evidence of a trend or seasonality. In Scotland, using daily data on calls to NHS24 about vomiting there was evidence of a weekly pattern and a trend (Wagner 2010). The data were best fitted by an AR(6) model on a seasonally differenced series. ARIMA models were used to describe the daily visits to emergency departments at a hospital in Boston over a period of 10 years from 1992 (Reis and Mandl 2003) and an $\operatorname{ARMA}(2,1)$ model was required for total visits, while an $\operatorname{ARMA}(1,1)$ model was required for respiratory visits.

An extensive investigation of the use of ARIMA modelling, in comparison to statistical process control methods (cf. Section 4) has been carried out (Williamson and Weatherby Hudson 1999). They found that ARIMA modelling was unable to model 8 out of 17 syndrome time series because of non-stationarity, partly arising from sparse data. Furthermore each series had to be investigated separately. For the series which were successfully modelled, one step ahead forecasts were satisfactory for forecasts up to 3 years in the future though better forecasts were obtained using continuously updated models.

In a syndromic-based detection approach, Reis and Mandl (2003) analyzed health care utilization patterns by a two-step time series modelling approach. A trimmed-mean seasonal model was used to capture both the yearly and weekly trends in daily utilization rates. The residuals from the trimmed-mean seasonal model were then fitted by an ARIMA model. An AR(6) model combined with a Serfling model with indicators variables for weekends and holidays was used to model attendance at ambulatory care centres for influenza like symptoms (Miller et al. 2003).

In these illustrations, the traditional ARIMA models require a relatively large number of parameters for the autocorrelation. Furthermore, a model the results for one syndrome is not easily adapted for use with another syndrome, so the whole process of model identification must be carried out each time, making the process difficult to automate.

With each new observation the parameters of the ARIMA model should be re-estimated. It is not clear how this can be achieved automatically, since model identification often relies on looking at residual plots. A practical approach may be to keep using the same model 
for a period of time, say one month for daily data and only refit the model each month. This might be practical for anyone series but not for many. Thus is is likely that ARIMA methods might be better suited to the retrospective analysis of time series data, rather than prospective use within an outbreak surveillance system.

Heisterkamp et al. (2006) proposed the use of a so-called hierarchical time series model, which does not require a long time series of data for parameter estimation. The observed counts are assumed to be Poisson distributed, whereas an unobserved process is assumed for the time trend in the expected number of cases. The models for the unobserved process range from a completely stationary process over time, to an autoregressive model of order 3. This provides a flexible model for the trend in a times series. Likelihood ratio tests are used to discriminate between the different models in the hierarchy. It is claimed that the hierarchical time series model is able to detect outbreaks faster than the Lab-Base exceedance system (Heisterkamp et al. 2006).

\subsection{Bayesian and hidden Markov models}

Le Strat and Carrat (1999) proposed the use of a hidden Markov model (HMM) (e.g., Cappé et al. 2005) for monitoring epidemiological data. The basic idea is to segment the time series of disease counts into an epidemic and non-epidemic phase. In the HMM of Le Strat and Carrat (1999) each observed $y_{t}(t=1, \ldots, n)$ is associated with a latent variable $z_{t} \in\{0,1\}$ that determines the conditional distribution of $y_{t}$. That is, $y_{t} \mid z_{t} \sim f_{k}\left(y_{t} ; \theta_{k}\right)$, where $k \in\{0,1\}, f_{k}$ is a pre-specified density (e.g. Gaussian or Poisson) and $\theta_{k}$ are parameters to be estimated. The unobserved state space, $z_{t}(t=1, \ldots, n)$ is modelled by a two-state homogeneous Markov chain of order 1 with stationary transition probabilities

$$
p_{k l}=\mathrm{P}\left(z_{t+1}=l \mid z_{t}=k\right),
$$

where $k, l \in\{0,1\}$ denote the two states of $z_{t}$ (1: epidemic; 0: non-epidemic). For example, $p_{01}$ is the probability of switching from the non-epidemic to the epidemic state. Note that in this Markov-dependent mixture model, $y_{t}$ is conditionally independent of all the remaining variables, given $z_{t}$. Le Strat and Carrat (1999) also considered HMMs with more than two hidden states and performed model selection using the Bayesian information criterion (BIC). Parameter estimates were obtained by means of a modified version of the EM algo- 
rithm (Dempster et al. 1977). Model extensions to account for time trends and seasonality were proposed using the cyclic regression function of Serfling (1963). An on-line version of the retrospective approach of Le Strat and Carrat (1999) is implemented in the R package surveillance (Höhle 2007; Höhle and Mazick 2010).

Rath et al. (2003) and Madigan (2005) presented further exploration of HMM for surveillance, the latter incorporating the Bayesian perspective, which requires prior distributions to be specified for model parameters. Markov Chain Monte Carlo (MCMC) methods were used for parameter estimation.

In another Bayesian approach, Martínez-Beneito et al. (2008) proposed a Markov switching model (e.g., Cappé et al. 2005) for prospective surveillance of weekly influenza incidence rates. In a Markov switching model the observed variables depend not only on the hidden state variables but also on the lagged observable variables. This setting makes the Markov switching model more suitable for time-series analysis than HMM. In Martínez-Beneito et al. (2008) the conditional distribution of the first-order difference series, formed by the differences between rates in consecutive weeks, is modelled either as a first-order autoregressive process or as a Gaussian white noise process, depending on whether the system is in an epidemic or non-epidemic phase. Let $y_{t, j}^{1}=y_{t+1, j}-y_{t, j}$ denote the first-order difference that corresponds to the difference between the rates in weeks $t+1$ and $t$ in season $j$. Conditional on the value of $z_{t}$,

$$
\begin{aligned}
y_{1, j}^{1} \mid z_{1}=0 & \sim \mathcal{N}\left(0, \sigma_{0, j}^{2}\right) \\
y_{1, j}^{1} \mid z_{1}=1 & \sim \mathcal{N}\left(0, \sigma_{1, j}^{2}\right) \\
y_{t, j}^{1} \mid z_{t}=0 & \sim \mathcal{N}\left(0, \sigma_{0, j}^{2}\right), \quad t \geq 2 \\
y_{t, j}^{1} \mid z_{t}=1 & \sim \mathcal{N}\left(\rho y_{t-1, j}^{1}, \sigma_{1, j}^{2}\right), \quad t \geq 2 .
\end{aligned}
$$

The advantage of using Markov switching models is that the differenced series is detrended, enabling autoregressive modelling to be used to analyse the data. The methodology described in Martínez-Beneito et al. (2008) is implemented in a web-based application called FluDetWeb (Conesa et al. 2009), which is used for the early detection of the onset of influenza epidemics. To illustrate this approach, we performed a prospective analysis using influenza-like illness (ILI) data from the Valencian Sentinel Network (VSN). The data set consists of eleven time series formed by the weekly ILI incidence rates (per 100,000 inhabitants in the Communitat 
Valenciana, Spain) during the seasons from 1996-1997 and 2006-2007. The data set can be downloaded via http://www.geeitema.org/doc/meviepi/influenza.html. An influenza season lasts 30 weeks (from the 42nd week of one year to the 19th week of the following year). In Figure 3, the weekly ILI incidence rates for the seasons 2005-2006 and 2006-2007 are compared. The black dots in Figure 3 (ii) indicate that the posterior probability of

(i)

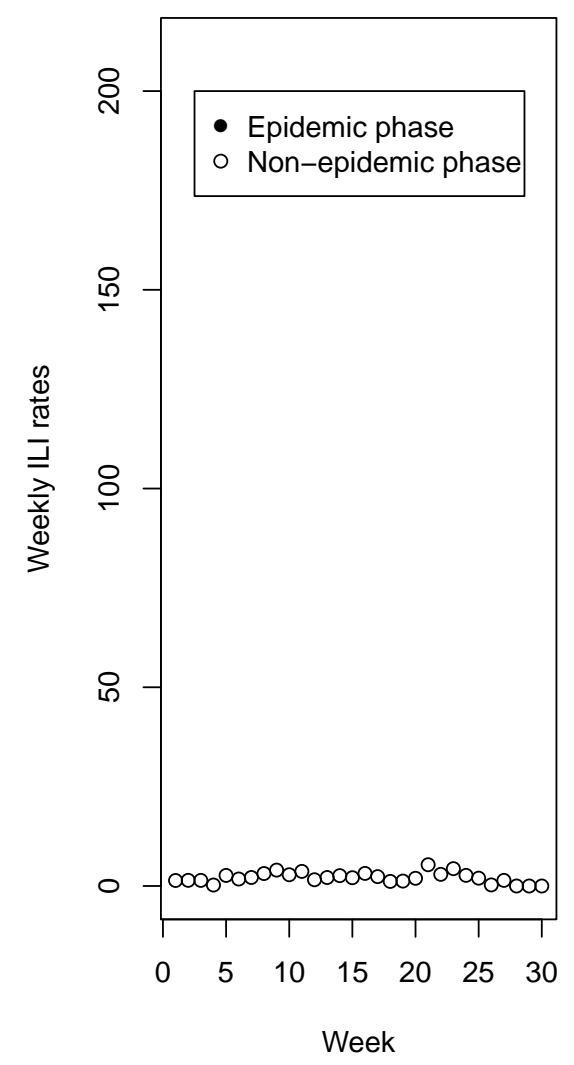

(ii)

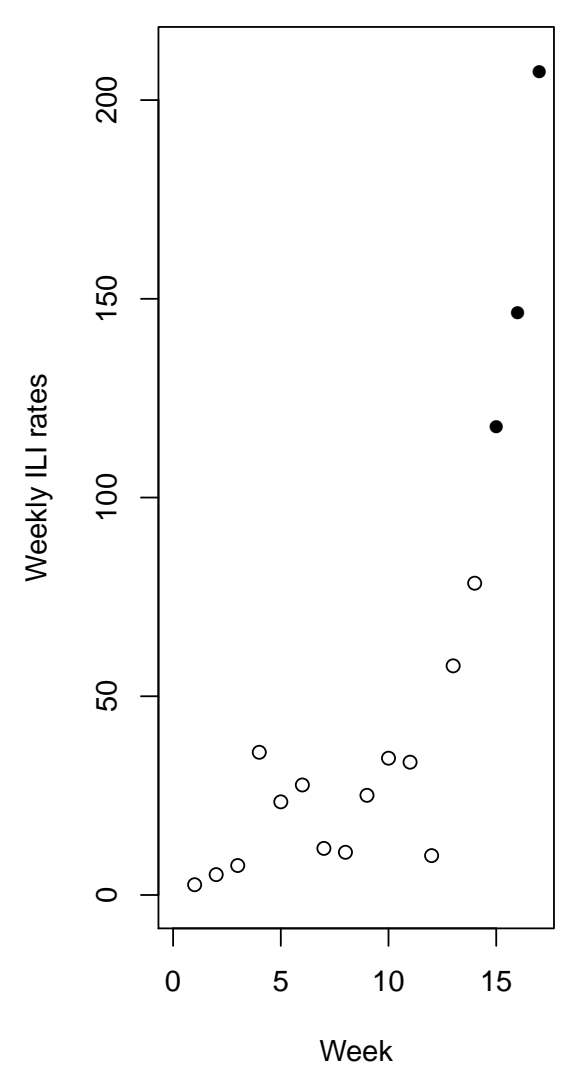

Figure 3: Comparison of weekly ILI incidence rates in the Communitat Valenciana, Spain, between the 2005-2006 (i) and 2006-2007 (ii) influenza season.

being in an epidemic phase exceeded 0.5 in week 15, 16 and 17 of the 2006-2007 influenza season. The posterior probabilities were found by means of the application FluDetWeb.

Conesa et al. (2010) introduced a framework of models with the idea of using them on any kind of surveillance data. In particular, the process of the observed cases is modelled via a Bayesian hierarchical Poisson model in which the intensity parameter is a function of the incidence rate. Different options for modelling the mean of the rates are described, including the option of modelling the mean at each phase as autoregressive processes of order 0,1 and 
2 (David Conesa 2010, personal communication).

Lu et al. (2010) developed a Markov switching model with jumps to handle the effect caused by past outbreaks. This model utilizes two additional hidden state variables in each period. The first hidden state variable models the disease outbreak state, and the second hidden state variable models the presence of extreme values. If an extreme value exists, the third hidden state variable represents the size of the extreme value. This is done to absorb any effect caused by sporadic extreme values in the training data.

In Cowling et al. (2006), dynamic linear models (West and Harrison 1997) were used as an approach to the early detection of the onset of the influenza epidemic period that requires only few weeks of baseline data.

For retrospective detection, Held et al. (2006b) proposed a two-component model where the counts are viewed as the sum of a 'parameter-driven' (endemic) and an 'observationdriven' (epidemic) component. The model for the parameter-driven component is a Poisson or negative binomial regression model. The observation-driven component is modeled with an autoregressive parameter. Model estimates are obtained through Bayesian inference via MCMC techniques. The retrospective approach of Held et al. (2006b) can be used in a prospective setting.

The above models have clear similarities in that they are designed to monitor a system that can exist in two states, where the time of the change from one state to another is unknown. The models can be generalized to three or more states but, in terms of their utility for outbreak surveillance, the need for more than two states is questionable, as the main aim is to detect a change. The main differences among the models is in their methods of modelling trend, seasonality and autocorrelation. For most of these models the simplest method of estimating the parameters is through Bayesian MCMC. While this is feasible for one infection or syndrome, computation time may present difficulties for surveillance with many endpoints where the models require daily updating.

\section{Methods inspired by statistical process control}

The methods of statistical process control (e.g., Montgomery 2009; Oakland 2008) have a long history of application to problems in public health surveillance (Woodall 2006). Sev- 
eral proposed approaches for the on-line detection of outbreaks of infectious diseases are directly inspired by, or related to, methods of statistical process control. This is not surprising because the problem of detecting unusual clusters of diseases in epidemiological data prospectively is similar to that of detecting aberrances in industrial production processes as they arise. The main tools for tracking the characteristics of a production process over time are control charts. These are discussed in Subsection 4.1. In Subsection 4.2 and Subsection 4.3, further methods are considered which share a flavor of the statistical process control methodology, namely temporal scan statistics and methods based on the time to failure.

\subsection{Control charts}

The first control chart for the mean of an independent Gaussian process was proposed by Shewhart (1931) (cf. Section 2). The Shewhart chart only utilizes information about the last time point. Later, Page (1954) and Roberts (1959) derived control charts with memory, the cumulative sum (CUSUM) and exponentially weighted moving average (EWMA) control chart, respectively. To start with the former, let $\left\{y_{t}, t=1,2, \ldots\right\}$ denote the time series of the counts being monitored. Assuming that $y_{t} \sim \mathcal{N}\left(\mu_{t}, \sigma_{t}^{2}\right)$, the one-sided (standardized) Gaussian CUSUM at time $t$ is defined iteratively by

$$
C_{t}=\max \left\{0, C_{t-1}+\left(\frac{y_{t}-\mu_{t}}{\sigma_{t}}-k\right)\right\},
$$

where $C_{0}=0$ and $k>0$ is a constant that depends on the size of the effect of interest. It is often chosen to be $1 / 2$ (Rogerson and Yamada 2004b). The baselines $\mu_{t}$ can be calculated from counts in comparable periods in previous years. These counts are also used to estimate the standard deviation $\sigma_{t}$. In the absence of any systematic departure from the expected values $\mu_{t}$, (11) remains at or close to zero. If $C_{t}>h$, where $h$ is a specified threshold value, the process is declared to be out-of-control. The CUSUM is then reset to zero and the process starts again. Figure 4 (ii) shows the CUSUM for Salmonella enteritidis in England, Wales and Northern Ireland in the year 2009. An outbreak from the 28th week onwards is detected by the CUSUM (11). The values in Figure 4 (i) are the weekly counts from the previous years 2000 to 2008 which were used to calculate $\mu_{t}$ and $\sigma_{t}$. The threshold $h$ was chosen on the basis of a predetermined acceptable value for the in-control average run length $\mathrm{ARL}_{0}$, i.e., the average time between alerts when there is no outbreak. The reciprocal of the 
(i)

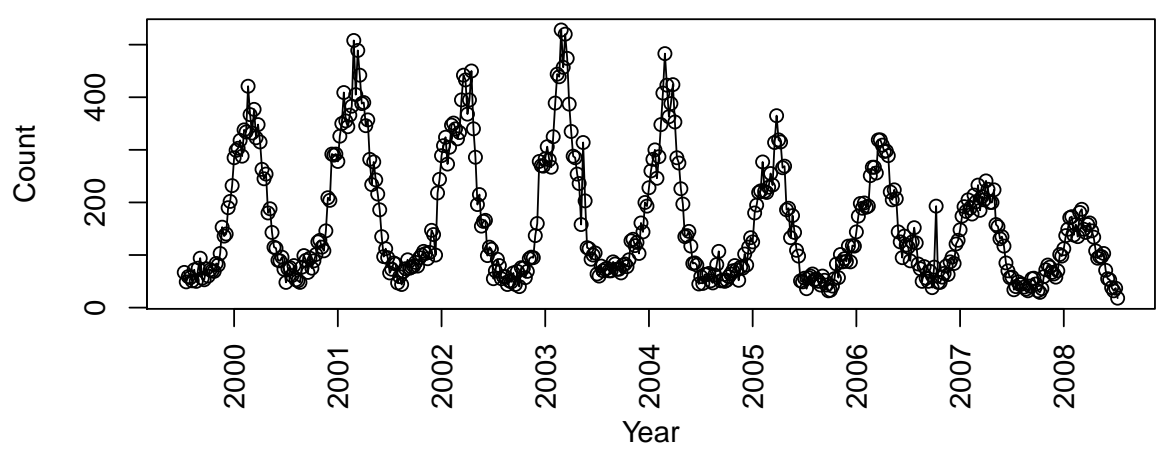

(ii)

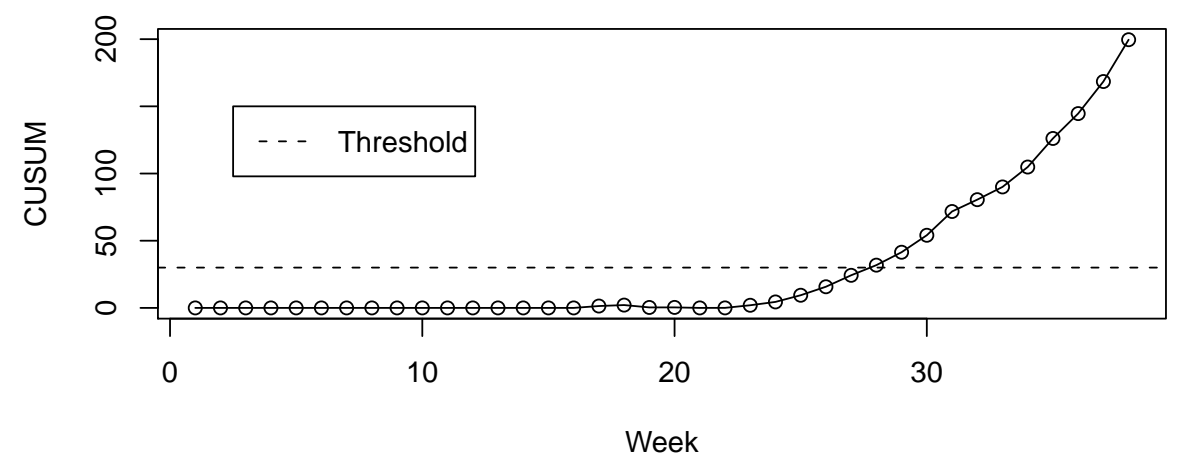

Figure 4: Weekly counts of Salmonella enteritidis PT 1, 4, 6, 6A, 8, 14B and 21 in England, Wales and Northern Ireland from 2000 to 2008 (i) and CUSUM of weekly counts in the year 2009 (ii) $(k=0.5, h=30)$.

$\mathrm{ARL}_{0}$ is the false positive (or false discovery) rate, i.e., the proportion of apparently aberrant reports not associated with outbreaks. Tables that can be used to find the value of $h$ that is associated with chosen values of $\mathrm{ARL}_{0}$ and $k$ are available (see for instance Rogerson 2001). In the case of rare events, the CUSUM approach (11) is not adequate, since the counts do not have a normal distribution. One remedy is to use the Poisson CUSUM (Lucas 1985). Other methods used in disease surveillance to detect an increase in the mean of a Poisson distribution include, for example, the short memory scheme of Shore and Quade (1989), which is based on the distribution of cases in the current and previous periods. Kenett and Pollak (1996) use the Shiryaev-Roberts statistic (Shiryaev 1963; Roberts 1966) and apply it to a non-homogeneous Poisson process. Whereas Gaussian or Poisson CUSUMs are designed to analyze counted data, binomial CUSUMs (e.g., Reynolds Jr. and Stoumbos 2000) can be 
used to monitor proportions.

Because CUSUMS are sensitive to small sustained changes in the mean numbers of reports, they are well suited to detecting the timing of onsets of relatively long-lasting epidemics, such as influenza. However, for the same reason, they are sensitive to small changes in reporting efficiency and other artefacts of the reporting process. Thus, they may lack robustness when used with surveillance data unless the baselines are frequently reset.

The EWMA control chart gives less weight to more historical data. The EWMA is defined by the following recursive equation:

$$
z_{t}=(1-\gamma) z_{t-1}+\gamma y_{t}
$$

where $z_{0}=0$ and the weight parameter $\gamma \in(0,1]$. The weighting for each older data point decreases exponentially, giving much more importance to recent observations, while not discarding older observations entirely. For $\gamma=1$, (12) is the same as the method by Shewhart (1931). The asymptotic (one-sided) variant of the EWMA chart will give an alarm at

$$
t_{A}=\min \left\{t: z_{t}>L \sigma_{z}\right\}
$$

where $L>0$ is a constant and $\sigma_{z}$ is the asymptotic standard deviation of $z_{t}$ (Sonesson 2003). Alternatively, one can use the exact standard deviation (which is increasing in time) instead of the asymptotic of the alarm limit (13). For the EWMA chart, Elbert and Burkom (2009) and Burkom et al. (2007) proposed the Holt-Winters technique for generalized exponential smoothing (cf. Subsection 3.1) to account for trends and seasonal features in syndromic data. Dong et al. (2008) constructed three types of EWMA methods that do not require an assumption of identical distributions of the counts to detect a positive shift in the incidence rate. Adaptions of the EWMA method for Poisson and binomial data are available (Borror et al. 1998; Gan 1991). Using the exponential smoothing technique and properties of numerical derivatives, Nobre and Stroup (1994) developed a method which bases monitoring on changes in the numerical gradient of the variable under surveillance with respect to time. Höhle and Paul (2008) present count data regression charts, which accommodate seasonal variation in the mean of the infectious disease counts. Assume that the observed counts originate from a negative binomial distribution parameterized by its mean $\mu$ and dispersion 
parameter $\theta$. Note that for $\theta \rightarrow 0$ the Poisson distribution with mean $\mu$ is obtained. For the in-control situation $y_{t} \sim N e g \operatorname{Bin}\left(\mu_{0, t}, \theta\right)$, where

$$
\log \left(\mu_{0, t}\right)=\alpha+\beta t+c(t)
$$

and $c(t)$ is a cyclic function that may be modelled, for example, by trigonometric terms (Serfling 1963). That is, the in-control mean is assumed to be time-varying and linear on the log-scale. The out-of-control situation is characterized by a multiplicative shift $\mu_{1, t}=\mu_{0, t} \times$ $\exp (\kappa)$ with $\kappa \geq 0$, which corresponds to an additive increase of the mean of the log-scale. It is assumed that the in-control parameters are known, while $\kappa$ is unknown and is estimated via maximum-likelihood. A generalized likelihood ratio statistic is computed to detect, on-line, whether a shift in the intercept occurred. Extensions of the basic seasonal count data regression chart are available that take account of autocorrelation between observations (Höhle and Paul 2008) or the population size of the age-strata (Höhle and Mazick 2010). Other modified CUSUM methods that allow for time-varying Poisson means were proposed by Rossi et al. (1999) and Rogerson and Yamada (2004a).

\subsection{Temporal scan statistics}

Scan statistics (e.g. Glaz et al. 2001) can be used to detect and evaluate clusters of disease cases in either a purely temporal, purely spatial or space-time setting (Woodall et al. 2008). In a temporal setting, this is usually done by gradually scanning a window across time, noting the number of observed and expected observations inside the interval. The scan statistic has long been used for retrospective detection of temporal clusters in epidemiology (Wallenstein 1980). Kulldorff (2001), Ismail et al. (2003) and Naus and Wallenstein (2006) adapted the scan statistic for the use in prospective temporal surveillance.

There are two general types of prospective temporal scan-based methods. One type involves counting the number of incidences in a single region in the most recent time period (or window) of a fixed length (Ismail et al. 2003; Naus and Wallenstein 2006). Let $y_{n}$ denote the observation at the current time point $n$ and let $L$ be the fixed window size. The scan statistic can be viewed as an unweighted moving sum (Han et al. 2010; Joner Jr. et al. 2008):

$$
S_{n}=\sum_{t=n-L+1}^{n} y_{t} .
$$


An alert is flagged as soon as (15) exceeds a threshold $h$, that is, the first time that $S_{n}>h$, where $h$ is chosen in conjunction with an acceptable value of $\mathrm{ARL}_{0}$.

In the prospective temporal scan method of Kulldorff (2001), the length of the window is not a constant but varies over a range of values. Since the temporal scan statistic by Kulldorff (2001) can be viewed as a special case of his spatio-temporal procedure, a discussion of this method is deferred till Section 5. Public health surveillance data are often non-stationary with seasonal and other effects that are seldom found in industrial process control data. Wallenstein and Naus (2004) proposed a temporal scan method that can account for seasonal effects.

\subsection{Methods based on inter-event times}

To model rare events, instead of monitoring aggregate total cases in discrete time periods, Chen (1978) proposed the so-called sets technique which bases detection on the intervals between reports. Devised for monitoring congenital abnormalities, in that context the sets technique is based on the number of healthy newborns delivered in the interval between the birth of an infant with the specific malformation and the birth of the next infant with that malformation. The group of births in one interval is named a set, and its size is a geometrically distributed random variable.

The sets technique may be applied to time intervals as well (e.g., Chen et al. 1997). Assuming that events arise in a homogeneous Poisson process, inter-event times follow an exponential distribution with mean $\mu$, say. The detection threshold is specified by parameters $(n, \tau)$, and an aberrance is declared if the intervals between $n+1$ consecutive events are all less than $\tau$. The false detection probability is $(1-\exp \{-\tau / \mu\})^{n}$, and the sensitivity of detection is $(1-\exp \{-\gamma \tau / \mu\})^{n}$ when the rate of events increases by a factor $\gamma$. The background rate, and hence $\mu$, may be determined from historical data. Appropriate values of $n$ and $\tau$ are selected to yield acceptable sensitivity and false detection probabilities. The analysis is performed whenever a new event occurs.

To reduce the expected frequency of false alarms obtained by the sets technique, Chen et al. (1993) suggested some confirmatory procedures. These declare an alarm as either confirmed or rejected according to data observed subsequent to the alarm. Reduction in the false 
detection probability is achieved at the expense of an increase in the delay in detecting a true alarm.

Note that methods which base detection on total reports will fail when events are very rare, because even a single report will then be unusual in a statistical sense. In such cases, one might either specify a minimum outbreak size that must be exceeded for the count to qualify as an aberration (Farrington et al. 1996), or alternatively use the sets monitoring technique. Noteworthy modifications of the sets technique are available: an extension proposed by Sitter et al. (1990, 1991) and the cumulative score procedure (Munford 1980; Wolter 1987). Sego et al. (2008) proposed the Bernoulli CUSUM chart for the surveillance of rare health events instead.

Other techniques based on the time to failure have been proposed, such as time-betweenevent (exponential) CUSUM or EWMA schemes (e.g., Gan 1994, 1998). Exponential control charts arise naturally in the context of monitoring the occurrence rate of rare events, since inter-event times for a homogeneous Poisson process are exponentially distributed random variables. The exponential CUSUM will detect a significant increase in the rate of incidence (corresponding to a decrease in the mean time between events) earlier than the Poisson CUSUM because, unlike the latter, it can trigger an alarm before the end of the time period under consideration.

\section{Methods incorporating spatial information}

\subsection{General}

As well as giving a date, in almost any surveillance system the reported incidence of a disease will specify a location. Using the spatial information given by the location can potentially enable small localised outbreaks of a disease to be detected. Ignoring this information, in contrast, will lead to the impact of a localised outbreak being diluted through its combination with global data, sometimes leading to its non-detection. In particular, localised outbreaks of common organisms will often be missed by surveillance systems that do not use spatial information. Other reasons for using spatial information can be the need to monitor the spread of an outbreak across a region, or interest may focus on changes in regional patterns 
of a disease and its outbreaks, or whether the disease occurs in clusters. Also, if a disease pattern is not uniform across the country then, preferably, the critical value at which an outbreak alarm is triggered should vary so as to reflect the underlying base-rate in each area.

To use spatial data, surveillance methods must have some notion of the distance between observations or some spatial structure. Several methods require only a cut-off value that categorises pairs of observations as either being 'close' or 'not close' (e.g., Rogerson 2001; Kulldorff 2001). In others, an appropriate distance metric is defined. Suppose that there are $m$ geographical units and let $\mathbf{S}$ be a $m \times m$ symmetric matrix of values $s_{k l}$ representing the closeness of geographical units $k$ and $l$, with $0 \leq s_{k l} \leq 1$. For example, Rogerson (1997) used the metric

$$
s_{k l}=\exp \left(-d_{k l} / \tau\right)
$$

where $d_{k l}$ is the geographic distance between reporting units $k$ and $l$ and $\tau$ is a specified constant. Tango (1995) suggested setting $\tau$ in (16) equal to five but results are fairly insensitive to its precise value (Bithell 1992). When the locations of observations are known individually, spatial structure has been imposed by using kernel estimation to fit a smooth surface that represents the intensity of reported cases (Diggle et al. 2005). Both fixed and variable kernel bandwidths have been used, but it seems sensible to have bandwidths that are narrower in large towns than in sparsely populated country areas. When a region is divided into sub-areas, spatial correlation can also be induced by defining a relationship between neighbouring areas. For example, if $u_{k}$ denotes the "location effect" of area $k$, then a common choice for modelling spatial correlation is to assume that $u_{k}$ follows the conditional autoregressive (CAR) model proposed by Besag et al. (1991). This model states that each $u_{k}$ is normally distributed around the mean value of $u$ amongst its immediate neighbours. That is, $u_{k} \mid\left\{u_{l}, l \neq k\right\} \sim \mathcal{N}\left(\bar{u}_{k}, \sigma^{2}\right)$, where

$$
\bar{u}_{k}=\sum_{l=1}^{m} u_{k} w_{k l} / \sum_{l=1}^{m} w_{k l}
$$

and

$$
w_{k l}= \begin{cases}1 & \text { if areas } k \text { and } l \text { are adjacent } \\ 0 & \text { otherwise }\end{cases}
$$


In principle, it is suboptimal to ignore spatial information in disease surveillance. Indeed, it may seem suboptimal to adopt any surveillance system that does not model the spacetime patterns of diseases as completely as possible when such information is available and reliable. However, the use of spatial information is computationally demanding, and it may not be practical for surveillance systems that monitor several hundred diseases and disease organisms - computer limitations will restrict the complexity of calculations that can be performed for each disease. Early approaches to the detection of localised disease outbreaks focused on only detecting outbreaks, enabling the methods to be comparatively simple. They include methods based on CUSUMs, and the scan statistic. These methods are described in Subsections 5.2 and 5.3, respectively. We then describe methods based on regression models in Subsection 5.4. These methods have the more ambitious aim of fitting space-time models to disease occurrences, typically using the Bayesian paradigm so that MCMC can be used as a tool to estimate model parameters. This can make the methods computationally very intensive. However, there is an example where regression methods have been applied in the routine surveillance of some specific diseases (e.g., Diggle et al. 2005), though strategies to reduce computation were necessary.

\subsection{Spatial CUSUM charts}

CUSUM charts are a standard approach for detecting outbreaks and changes in spatial pattern. CUSUMs cumulate information from each case. In the spatial context, the information cumulated typically involves spatio-temporal proximity to other points. In order to isolate the specific contribution of the latest observation, conditioning on previous information is required. Thus the formula for the CUSUM given in equation (11) must be modified. The modified formula is

$$
C_{t}=\max \left\{0, C_{t-1}+\left(\frac{y_{t}-\mathrm{E}\left(y_{t} \mid \nu_{t-1}\right)}{\operatorname{var}\left(y_{t} \mid \nu_{t-1}\right)^{1 / 2}}-k\right)\right\},
$$

where $C_{t}$ is the CUSUM at time $t, k$ is a specified constant, and $\mathrm{E}\left(y_{t} \mid \nu_{t-1}\right)$ and $\operatorname{var}\left(y_{t} \mid \nu_{t-1}\right)$ are the expectation and variance of $y_{t}$ conditional on relevant information $\nu_{t-1}$ from the first $t-1$ reporting periods, respectively. An alarm is flagged if the CUSUM exceeds a threshold. Statistics developed for the retrospective analysis of spatial disease data have been adapted to give the $y_{t}$. The Knox test (Knox 1964) aims to identify space-time interactions by 
categorising any two cases as close in space, close in time, close in both, or close in neither. The number of observations that are close in both space and time is referred to as the Knox statistic and a space-time interaction is indicated if it is unusually large. Rogerson (2001) adapted the Knox statistic for use in surveillance by equating its value after $t$ cases to $y_{t}$ in the CUSUM in equation (17). He showed that $\nu_{t-1}$ may be replaced by $y_{t-1}$ (i.e. $y_{t-1}$ contains all the relevant information in the first $t-1$ observations). He also gave formulas for the conditional means and variances needed to form the CUSUM. However, empirical testing by Marshall et al. (2007) suggests that these estimates of means and variances can be poor and that computer simulation should be used to estimate both them and the threshold at which an alarm is triggered.

Rogerson (1997) adapted Tango's statistic (Tango 1995) in a way similar to the method used with the Knox statistic. Tango's statistic for spatial clustering has the form

$$
y=(\mathbf{r}-\mathbf{p})^{\top} \mathbf{S}(\mathbf{r}-\mathbf{p})
$$

where $\mathbf{r}$ and $\mathbf{p}$ are $m \times 1$ vectors containing the observed and expected proportions of cases at each location, respectively, and $\mathbf{S}$ has the meaning assigned in Subsection 5.1. The statistic (18) was designed as a retrospective test of spatial clustering and is completely insensitive to a global change in the rate of disease occurrence. In part this is a disadvantage, but it also means that the statistic remains valid when there is seasonality and/or annual trend. Raubertas (1989) gave a method that forms neighbourhoods in a way that allows a reporting unit to belong to more than one neighbourhood. The data within a neighbourhood are pooled in weighted averages, using a measure of closeness as weights. For each neighbourhood a CUSUM is formed that monitors incidence rate and an alarm is triggered if any CUSUM exceeds a threshold. This early method continues to influence surveillance methods in varied contexts (e.g., Sparks 2010).

ClusterSeer (Jacquez et al. 2002) and GeoSurveillance (Yamada et al. 2009) are software packages that implement surveillance methods based on spatial CUSUMs. The GeoSurveillance package was used to perform spatio-temporal surveillance of the weekly counts of all Salmonella enteritidis cases in England, Wales and Northern Ireland in 2009. The geographical units are twelve regions defined by the HPA, namely "North East", "Yorkshire \& Humberside", "East Midlands", "East", "London", "South East", "South West", "West 
Midlands", "North West", "Channel Islands \& Isle of Man", "Wales" and "Northern Ireland". Figure 5 displays the results for four of these regions and gives evidence that spatial heterogeneity is inherent in the data (see also Figure 4 in which data aggregated over the twelve geographical areas are presented).

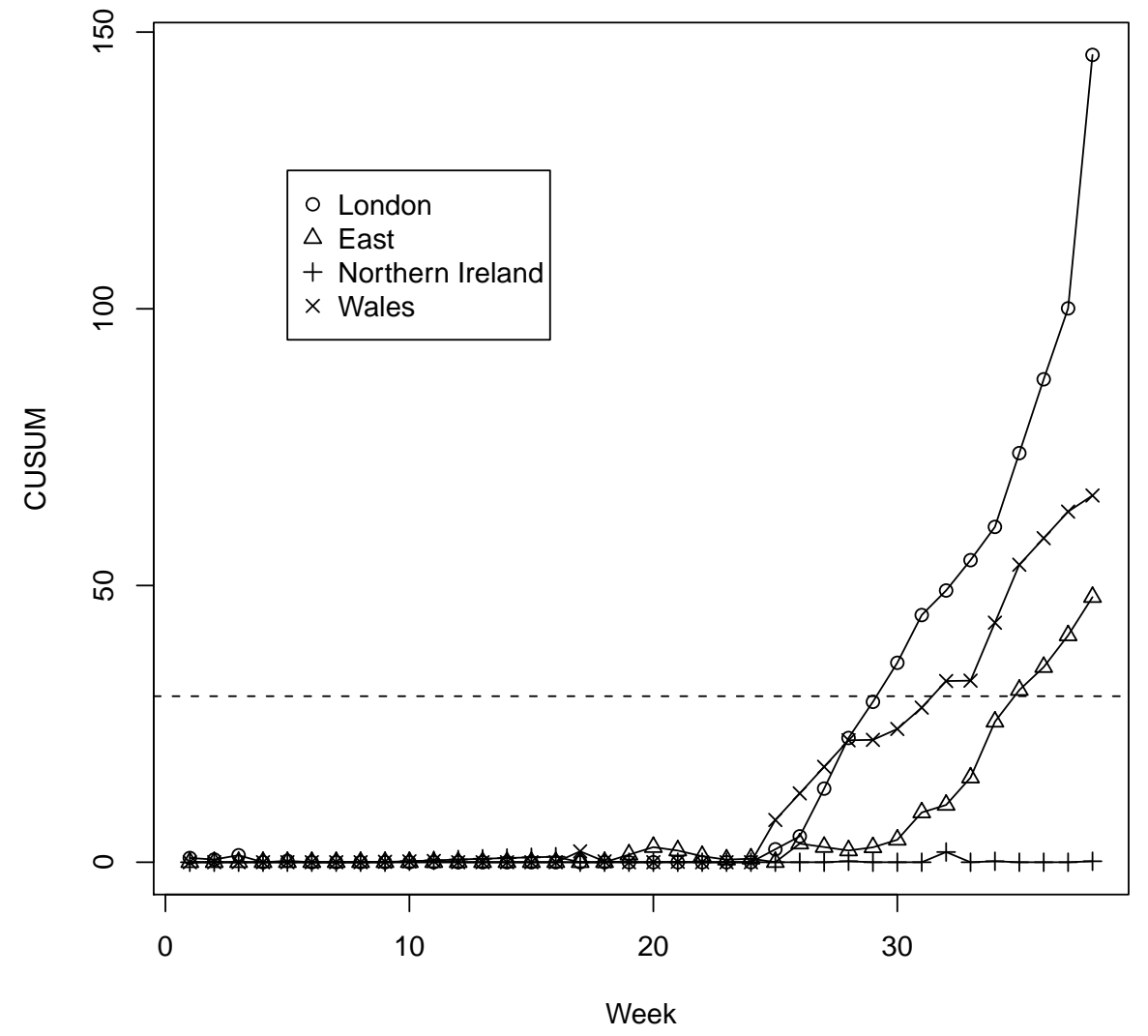

Figure 5: CUSUMs of weekly counts of Salmonella enteritidis PT 1, 4, 6, 6A, 8, 14B and 21 for four regions in England, Wales and Northern Ireland in the year 2009.

\subsection{Space-time scan statistics}

For a temporal scan, a search window is gradually moved across time, looking for a window in which the number of observed cases is unexpectedly high (cf. Subsection 4.2). Similarly, for a spacial scan, a circular window is moved over a map of the study area, looking for a position where the circle contains an unexpectedly high number of cases. For a space-time scan the two are combined to form a cylindrical 'window' whose height is the time-dimension. 
This form of search window has been used for the retrospective detection of local disease outbreaks since at least Wallenstein et al. (1989). Kulldorff (2001) adapted the method for prospective surveillance.

The temporal component of the scan windows used by Kulldorff (2001) has a varying start time but reaches to the end of the current monitoring period, as the aim is to detect local disease outbreaks that are currently active. The spatial components of the windows have varying centres and radii. A search finds the scan window that has the most unexpectedly high number of cases, judged by the likelihood ratio

$$
L R=\frac{L(z)}{L_{0}}
$$

where $L(z)$ is the maximum likelihood for cylinder $z$ if the scan window has its own occurrence rate and $L_{0}$ is the maximum likelihood if the scan window has the underlying base rate as its occurrence rate. The likelihoods in (19) are generally based on the assumption that the number of cases follows a Poisson distribution. The maximum likelihood ratio is defined to be the space-time scan statistic and a $p$-value for its significance is obtained through Monte Carlo hypothesis testing.

Various methods aimed at improving the scan statistic have been suggested. Kleinman et al. (2005) focused on the baseline rates of disease incidence in the population-at-risk, which are used in calculating the scan statistic. They proposed one estimate of these rates that incorporates an adjustment for day-of-week, month and holidays, and a second estimate that includes an additional adjustment for local history of illness. Kulldorff et al. (2005) suggested a scan statistic that does not require the size of the population-at-risk to be estimated and only needs data on cases. This scan statistic was defined as the minimum $p$-value from testing for a space time interaction in a scan window. Its assumptions are similar to those made with the Knox statistic. Assunção and Correa (2009) adapt the Shiryaev-Roberts statistic (Shiryaev 1963; Roberts 1966) as a scan statistic, modelling a disease outbreak as a change point in a cylindrical scan window. They improve the computation speed of their method through formula for updating estimates when a case occurs, rather than having to calculate estimates from scratch.

Sonesson (2007) fits the space-time scan statistics of Kulldorff (2001) and Kulldorff et al. (2005) into a CUSUM framework and examines properties of the resultant methods. Spatial 
scan statistics (without a temporal component) have also been used for disease surveillance by considering case incidence in a short fixed time period, such as the last seven days (Mostashari et al. 2003). In a different direction, using ellipses (rather than circles) for the spatial component of a space-time scan window has been suggested (Kulldorff et al. 2006). Also, non-parametric spatial scan windows to detect irregularly shaped clusters have also been explored (Duczmal and Assunção 2004; Tango 2005; Assunção et al. 2006). However using such windows significantly increases computer time.

The space-time scan statistic is currently the most widely used method for detecting the emergence of localised disease clusters (Shmueli and Burkom 2010). In one application it was used with a dataset on lower respiratory disease in Boston. The work showed the importance in some circumstances of modifying base rates to allow for seasonal effects and day-of-the-week effects - otherwise there appeared to be significant clusters on most weekdays between October and February (Kleinman et al. 2005). In another application the spacetime scan statistic proved better at detecting emerging clusters of male thyroid incidence in New Mexico than a scan statistic that was purely spatial (Kulldorff 2001). Kulldorff et al. (2005) demonstrated the effectiveness of their method with data on diarrhea and influenza in New York City: four of the five clearest alarms given by their method were likely local precursors of city-wide outbreaks and the number of false alarms was modest. Space-time scan statistics have also been used with data on Burkitt's lymphoma (Assunção and Correa 2009), brain cancer (Kulldorff et al. 1998), and tularemia (Sonesson 2007).

SaTScan (Kulldorff 2010) and ClusterSeer (Jacquez et al. 2002) are software packages that implement scan statistic methods. SaTScan is used by various American federal, state and city agencies for retrospective and prospective cluster detection (Zhang and Lin 2009), including the New York City Department of Health, who use it for syndromic surveillance.

\subsection{Spatio-temporal regression methods}

A variety of spatio-temporal regression methods have been proposed. An important distinction between them is whether they analyse aggregated data or data at an individual level. Often the region of interest is broken into small areas and the aggregated number of cases in each area in each time period forms the response. This form of area-level data has usually 
been modelled as a discrete spatial model in which neighbourhood relationships between the areas are defined. The second form of data can arise with sparse data, when the location of each case is recorded at an individual level. These individual-level data have been modelled as a Cox point process (Cox 1955).

For an area-level model, let $y_{k t}$ denote the number of cases in area $k$ in time period $t$. Sometimes the model specifies that $y_{k t}$ follows a binomial distribution (e.g., Diggle et al. 2004; Kleinman et al. 2004) but, more commonly, it is assumed that $y_{k t}$ follows a Poisson distribution. Interest then centres on estimating the Poisson mean (Lawson et al. 2003; Vidal Rodeiro and Lawson 2006; Watkins et al. 2009; Zhou and Lawson 2008), which varies with $k$ and $t$. Lawson and his co-workers generally assume that $\mathrm{E}\left(y_{k t}\right)=e_{k t} \theta_{k t}$, where $\theta_{k t}$ is the unknown true relative risk in area $k$ and time period $t$ and $e_{k t}$ is the expected number of cases in the $k$ th area in that period. The values of $e_{k t}$ must be specified before the model is fitted or else there is an identifiability problem. The $e_{k t}$ could be based on the "at risk" population demographics in each area, perhaps with an adjustment for seasonality or trend. Information about the $e_{k t}$ might also be gleaned from monitoring a different disease that has a similar at-risk population structure to the disease of interest (Lawson 2005). The logarithm of the relative risk is decomposed into spatio-temporal components:

$$
\log \theta_{k t}=u_{k}+v_{k}+\tau_{t}+\gamma_{k t},
$$

where $u_{k}$ represents spatially correlated extra variation, $v_{k}$ represents uncorrelated extra variation, $\tau_{t}$ describes temporal variation, and $\gamma_{k t}$ is the space-time interaction. It is generally assumed that $\tau_{t}$ and $\gamma_{k t}$ follow random walks to allow a smooth variation in time (Knorr-Held 2000): $\tau_{t} \sim \mathcal{N}\left(\tau_{t-1}, \sigma_{\tau}^{2}\right)$ and $\gamma_{k t} \sim \mathcal{N}\left(\gamma_{k, t-1}, \sigma \gamma^{2}\right)$. In general, the CAR model of Besag et al. (1991) is used to model the spatial correlation between the $u_{k}$.

The complexity of this model makes it virtually essential to use Bayesian methods for model fitting. Vague prior distributions are given to the model parameters and MCMC methods are used to sample from the posterior distribution and estimate parameters. To test for changes in spatial pattern, the estimates of parameters could be monitored (Lawson et al. 2003) but, much more commonly, observations are compared with one-step-ahead predictions (Kleinman et al. 2004).

Other modelling stategies have also been proposed. Zhou and Lawson (2008) give a compu- 
tationally cheap approach in which separate spacial models are fitted to the data from each collection period. These models are combined by forming exponentially weighted moving averages and a sharp change in the weighted average in any neighbourhood suggests an outbreak. Another computationally quick method is given by Kleinman et al. (2004), who fit generalised linear mixed models that include time-of-day and seasonality components, but which only allow uncorrelated heterogeneity between areas. Several researchers have used a model in which the disease process can switch between two (unobserved) states: endemic (non-outbreak) and epidemic (outbreak). To model the process HMMs are used and an alarm is flagged on the basis of a Bayes factor that reflects the relative likelihoods of each state (Lawson et al. 2003; Madigan 2005; Watkins et al. 2009).

Modelling individual-level data has attracted far less attention. Clark and Lawson (2006) proposed a novel approach using non-parametric regression via kernel smoothers. However the major work with individual-level data was in the AEGISS (Ascertainment and Enhancement of Gastrointestinal Infection Surveillance and Statistics) project, reported in Diggle et al. (2004) and Diggle et al. (2005). The aim of this project was to develop a monitoring tool that could identify anomalies in the space-time distribution of non-specific gastrointestinal infections. The data it collected was the location, $x$, and date, $t$, of each individual case. As the point process model for these data, Diggle et al. (2005) used a non-stationary log-Gaussian Cox process in which the spatio-temporal intensity, $\lambda(x, t)$ was decomposed as

$$
\lambda(x, t)=\lambda_{0}(x) \mu_{0}(t) R(x, t)
$$

where $\lambda_{0}(x)$ is a smoothly varying surface describing the normal disease pattern that was estimated using kernel smoothing, $\mu_{0}(t)$ is temporal variation, modelled parametrically to reflect day-of-week and season effects, and $R(x, t)$ is the residual space-time variation.

Both $\lambda_{0}(x)$ and $\mu_{0}(t)$ in (20) could be estimated from historical data but up-to-date predictions of $R(x, t)$ were required. These predictions were based on the most recent five days data. Naturally they had far more uncertainty attached to them than the estimates of $\lambda_{0}(x)$ and $\mu_{0}(t)$, so these latter estimates were treated as deterministic quantities. To detect outbreaks, the region was divided into neighbourhoods and MCMC was used to determine the probability in each neighbourhood that $R(x, t)$ exceeded an agreed threshold. The results were reported daily on maps with colours denoting probability levels. 
The complexity of models places a burden on computer resources and the problem is exacerbated if MCMC is used to implement Bayesian methods. As noted by Lawson et al. (2004, p. 952), .. for any Bayesian model, computational speed-ups must be sought to make implementation realistic in a surveillance context. In line with this, it seems sensible to follow Diggle et al. (2005) and treat any quantity that is estimated from historical data as deterministic. Also, historical data can generally be restricted to a moving window of the last 3, 5 or 8 years (Lawson et al. 2003) unless the data are very sparse. Similarly, a window of just the most recent past (perhaps only a few days) has generally been used when much more weight should be given to recent data (e.g., Diggle et al. 2005). Numerical approximations have also been used to good effect. For example, Kleinman et al. (2004) estimated parameters through quasi-likelihood, which is computationally less demanding but which introduces some bias. In many situations these pragmatic measures would simply be an option, but in the context of real-time surveillance they are a necessity.

\section{Multivariate outbreak detection}

\subsection{Scope of multivariate detection}

Most outbreak detection systems track more than one data series. For example, in the UK, systems of laboratory surveillance (Farrington et al. 1996; McCabe et al. 2003), syndromic surveillance (Baker et al. 2003; Robertson 2006), and systems for institutional surveillance (Marshall et al. 2004) may typically monitor dozens or even hundreds of different data series. When the different data series (and, most importantly, outbreaks within them) are likely to be unrelated, it usually makes most sense to consider them as separate univariate series. However, in some cases, several series will relate to the same underlying process, and hence process changes are likely to be strongly correlated. This applies, for example, to indicators of influenza (Stroup et al. 1988; Griffin et al. 2009; Mann 2009), reports of gastrointestinal illness from different sources (Kulldorff et al. 2007), or time series of counts of the same infection in different age groups (Held et al. 2005). In such circumstances multivariate methods of outbreak detection are likely to be fruitful in exploiting dependencies, both between the underlying processes and between the timing of outbreaks. 
An overview of methods for multivariate surveillance is given in Sonesson and Frisén (2005) and Frisén (2010), who classified genuinely multivariate approaches into categories that include reduction of dimensionality, joint modelling, and vector accumulation methods. These are discussed in turn in Subsection 6.1, Subsection 6.2 and Subsection 6.3, respectively. Sonesson and Frisén (2005) and Frisén (2010) also mention so-called 'parallel surveillance' methods. A parallel approach monitors each variable separately by means of a univariate surveillance method. An alarm for the multivariate process is declared if some condition is fulfilled, e.g. the first time that any of the univariate processes gives an alarm. These methods will not be considered further here.

\subsection{Dimension reduction}

Dimension reduction methods for multivariate surveillance data could in principle include standard tools such as principal component analysis (Jolliffe 2002). These have been used for detecting aberrations in other fields ( $\mathrm{Ku}$ et al. 1995), though they may lead to problems of interpretation. A more popular approach is to reduce the multivariate data at each time point to a scalar, which is then monitored by univariate surveillance methods. Let $\mathbf{y}=\left\{\mathbf{y}_{t}, t=1,2, \ldots\right\}$ be the multivariate process under surveillance, where $\mathbf{y}_{t}=$ $\left(y_{1 t}, y_{2 t}, \ldots, y_{p t}\right)^{\top}$ is observed with in-control mean $p \times 1$ vector $\boldsymbol{\mu}$ and $p \times p$ covariance matrix $\boldsymbol{\Sigma}$. An early multivariate surveillance scheme is that based on Hotelling's $T^{2}$ (Hotelling 1947; Jackson 1959). The process parameter at time $t$ for multivariate data $\mathbf{y}_{t}$ is

$$
T^{2}(t)=\left(\mathbf{y}_{t}-\boldsymbol{\mu}\right)^{\top} \boldsymbol{\Sigma}^{-1}\left(\mathbf{y}_{t}-\boldsymbol{\mu}\right)
$$

The statistic (21) is a multivariate extension of the Shewhart chart, an alarm being declared if $t_{A}=\min \left\{t: T^{2}(t)>h\right\}$, where $h$ is a specified threshold. For a bivariate process $\left(x_{t}, y_{t}\right)$ with changepoint $\left(t_{x}, t_{x}\right)$, Andersson $(2009 \mathrm{~b})$ shows that the conditional expected delay, defined as $t_{A}-t_{(1)}$ given that $t_{A}>t_{(1)}$, where $t_{(1)}=\min \left\{t_{x}, t_{y}\right\}$, depends only on $\left|t_{x}-t_{y}\right|$. A further possibility is to undertake univariate analyses on each data set, and combine the $p$-values for the marginal tests into a single 'consensus' value. One such method uses Fisher's rule to obtain the summary statistic $F$ from $n$ individual $p$-value $p_{i}$ :

$$
F=2 \sum_{i}^{n} \ln \left(p_{i}\right) \text {. }
$$


If the $n$ tests are independent then the null distribution of $F$ is $\chi^{2}$ with $2 n$ degrees of freedom. This and other methods for combining $p$-values, along with Hotelling's $T^{2}$, are discussed in an outbreak detection setting by Burkom et al. (2005).

\subsection{Joint modelling methods}

Kulldorff et al. (2007) developed a multivariate space-time scan statistic based on the sum of the log likelihood ratio statistics for the univariate processes. This generalizes an earlier univariate version (Kulldorff 1997). Thus, suppose that the total number of reports for series $j$ is $N_{j}(j=1, \ldots, p)$. For a space-time cylinder $z$ with $n_{j, z}$ cases from series $j$ and expected cases $\mu_{j, z}$ obtained under a Poisson model, the likelihood ratio for a 'high' cluster is

$$
L R_{j}(z)=\left(\frac{n_{j, z}}{\mu_{j, z}}\right)^{n_{j, z}}\left(\frac{N_{j}-n_{j, z}}{N_{j}-\mu_{j, z}}\right)^{N_{j}-n_{j, z}}
$$

if $n_{j, z}>\mu_{j, z}$ and 1 otherwise. The multivariate scan statistic for detecting 'highs' is then

$$
T=\max _{z} \sum_{j} \log L R_{j}(z)
$$

which adjusts automatically for multiple testing inherent in considering multiple series as well as multiple cylinders. Note that equation (22) differs very slightly from Kulldorff's formulation: his $L R_{j}(z)$ is multiplied by an indicator function, but this will cause problems when taking logarithms.

To illustrate this approach, the multivariate space-time scan statistic (23) was applied to syndromic surveillance data in Scotland. The data give the number of calls to NHS24 by postcode district and day from 19 February to 1 April 2007 originated within the Glasgow (the ' $G$ ') postcode area (see Figure 6). This area consists of 50 postcode districts. Data are recorded for the two symptoms diarrhoea and vomiting which are syndromic indicators for norovirus infection. A space-time scan statistic analysis was performed for each of the 7 days from 26 March to 1 April, using the data from 19 February 2007. We used 3 days as the maximum temporal window size. Calculations were performed using the SaTScan software (Kulldorff 2010). The results of the analyses are presented in Table 1. The strongest signal was on 1 April, with the cluster consisting of 2 days and 25 postcode districts. With a recurrence interval of 2 years and 269 days, this cluster is unlikely to be a chance occurrence. 


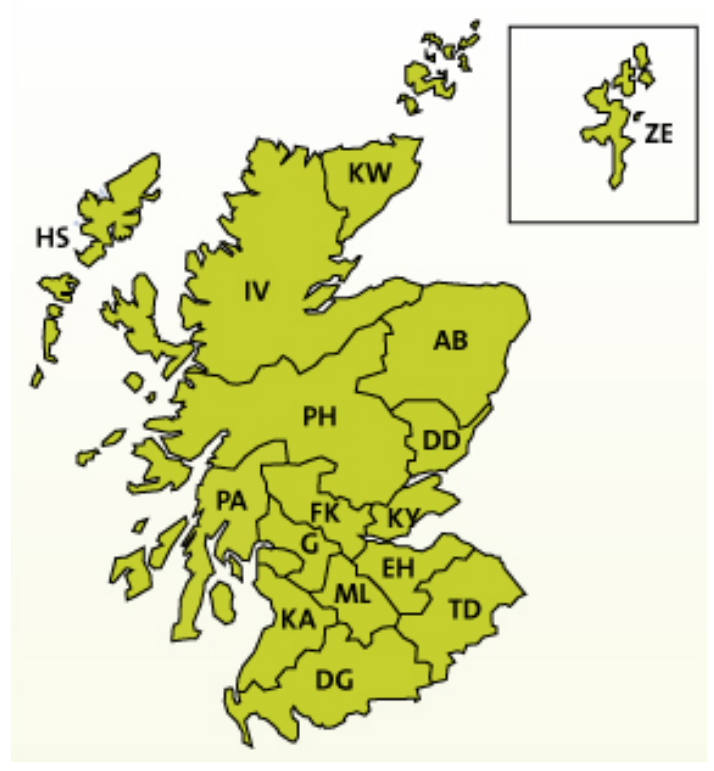

Figure 6: Map of Scotland with 'G' (Glasgow) postcode area.

Table 1: The two most likely clusters for norovirus in the Glasgow postcode area, during 26 March to 1 April 2007, as generated by the multivariate space-time scan statistic (Obs. (observed), Exp. (expected), RR (relative risk), RI (recurrence interval)).

\begin{tabular}{cccccccccc}
\hline \multicolumn{2}{c}{ Cluster characteristics } & \multicolumn{9}{c}{ Diarrhoea } & \multicolumn{1}{c}{ Vomiting } \\
Date & \#postc. & \#days & Obs. & Exp. & RR & Obs. & Exp. & RR & RI \\
\hline 26 March & 27 & 3 & 49 & 49.29 & 0.99 & 192 & 123.84 & 1.59 & 2 years 269 days \\
1 April & 25 & 2 & 47 & 28.28 & 1.69 & 164 & 76.02 & 2.22 & 2 years 269 days \\
\hline
\end{tabular}

A second cluster with an identical recurrence interval was detected on March 26, containing 27 postcode districts during 3 days.

Other joint modelling approaches have been suggested, based on a joint model for the entire multivariate data series. In one such method, the alarm function is based on the likelihood ratio derived from the joint distribution of the multivariate process (Andersson 2009a). This is a multivariate version of the method of Frisén and de Maré (1991). Schiöler and Frisén (2010) present a multivariate extension of the semiparametric univariate method of Frisén and Andersson (2009), based on the sufficient reduction approach of Frisén et al. (2010b) for step changes.

Several other joint modelling methods have been used for infectious disease data, but have not been applied to outbreak detection per se, though extensions in this direction should 
be possible. Held et al. (2005) extended to the multivariate case a model incorporating a branching process presented in Held et al. (2006b). This model was further extended in Paul et al. (2008) to analyse data from several pathogens. A multivariate spatial model for different gastrointestinal infections was presented in Held et al. (2006a).

Sebastiani et al. (2006) used dynamic Bayesian networks to study the interplay of four different data sources that are monitored for influenza surveillance. Mann (2009) developed a multivariate HMM with a shared hidden process to model several markers of influenza.

\subsection{Vector accumulation methods}

Vector accumulation methods include multivariate extensions of the EWMA and CUSUM charts, referred to as MEWMA and MCUSUM, respectively. Generalizing the EWMA is relatively straightforward (Lowry et al. 1992), via the recursive scheme

$$
\mathbf{z}_{t}=\boldsymbol{\Lambda}\left(\mathbf{z}_{t}-\boldsymbol{\mu}\right)+\left(\mathbf{I}_{p}-\boldsymbol{\Lambda}\right) \mathbf{z}_{t-1}
$$

where $\boldsymbol{\Lambda}$ is a diagonal $p \times p$ matrix of values in $(0,1]$ and $\mathbf{I}_{p}$ is an identity matrix of order $p$. The chart from (24) goes out of control when

$$
T_{t}^{2}=\mathbf{z}_{t}^{\top} \boldsymbol{\Sigma}_{\mathbf{z}_{t}}^{-1} \mathbf{z}_{t}>h
$$

for some control limit $h$, where $\boldsymbol{\Sigma}_{\mathbf{z}_{t}}$ is the covariance matrix of $\mathbf{z}_{t}$. In contrast, generalising the standard univariate two-sided CUSUM is complicated by the fact that there are two cumulative sums for each variable. Crosier (1988) and Pignatiello Jr. and Runger (1990) developed multivariate CUSUMS as generalisations of new univariate CUSUMS requiring a single cumulative sum per variable. Golosnoy et al. (2009) investigated the properties of the MCUSUM chart of Pignatiello Jr. and Runger (1990) and suggested further enhancements. Ngai and Zhang (2001) generalized the standard univariate CUSUM in the sense that the $p$-dimensional version reduces to it when $p=1$. These various MCUSUMS all apply only to independent multivariate observations. Bodnar and Schmid (2007) further extended these methods to multivariate time series, taking into account dependencies in the underlying process. Moving beyond Gaussian processes, an example of a rank-based MCUSUM can be found in Qiu and Hawkins (2001), with further development of this non-parametric scheme provided by Qiu and Hawkins (2003). 


\section{$7 \quad$ Final remarks}

In this paper we have sought to review the methods that have been proposed for identifying outbreaks of infectious diseases as they arise, in sufficiently timely fashion to allow interventions to take place. In limiting ourselves to these statistical methods, we chose not to deal with several issues of critical importance, which require further research and involvement of statisticians. One key issue is how to evaluate statistical surveillance systems, especially in the absence of a gold standard. Another is how to design effective and flexible user interfaces - ideally including a choice of statistical algorithms. A third is how to create effective protocols to handle the signals that emerge from a statistical surveillance system. For systems tracking many different organisms, this includes controlling the false discovery rate (Benjamini and Hochberg 1995). A fourth is how to deal with the data imperfections, such as reporting delays, which inevitably affect prospective detection systems.

This review shows that a very broad range of statistical techniques have been proposed for prospective outbreak detection. They range from the very simple to the very complex, involve both testing and modelling approaches, and increasingly make use of modern simulation-based inference, often in a Bayesian framework. The choice of which statistical technique to use will depend critically on the nature of the intended application. In particular, systems designed for the surveillance of a single infection or syndrome should arguably be tuned to the specific features of that infection and may need frequent user intervention. In contrast, systems designed for routine application to hundreds or thousands of possible infections, with diverse frequencies and temporal patterns, will require robustness and automation. No single statistical technique is likely to be ideal in every setting.

Statistical outbreak detection is a multidisciplinary science, involving epidemiologists and computer programmers, as well as statisticians. Its rapid development over recent years presents an opportunity for statisticians, through the numerous techniques now available, and the increased acceptance of statistical methodology in this field. But it also presents a challenge to the statisticians: to demonstrate that these new statistical methodologies provide added value in public health terms, by effectively supplementing other surveillance methods for detecting outbreaks. 


\section{Acknowledgements}

This study was supported by grants from the National Institute for Health Research and Medical Research Council. We are grateful to David Conesa, Marianne Frisén, Michael Höhle and Ron Kenett for making us aware of some references that we would otherwise have missed.

\section{References}

Andersson, E. (2009a), "Effect of dependency in systems for multivariate surveillance," Communications in Statistics - Simulation and Computation, 38, 454-472.

— (2009b), "Hotelling's $T^{2}$ method in multivariate on-line surveillance: on the delay of an alarm," Communications in Statistics - Theory and Methods, 38, 2625-2633.

Andersson, E., Bock, D., and Frisén, M. (2008), "Modeling influenza incidence for the purpose of on-line monitoring," Statistical Methods in Medical Research, 17, 421-438.

Assunção, R. and Correa, T. (2009), "Surveillance to detect emerging space-time clusters," Computational Statistics \& Data Analysis, 53, 2817-2830.

Assunção, R., Costa, M., Tavares, A., and Ferreira, S. (2006), "Fast detection of arbitrarily shaped disease clusters," Statistics in Medicine, 25, 723-742.

Baker, M., Smith, G. E., Cooper, D., Verlander, N. Q., Chinemana, F., Cotterill, S., Hollyoak, V., and Griffiths, R. (2003), "Early warning and NHS Direct: a role in community surveillance?" Journal of Public Health Medicine, 25, 362-368.

Benjamini, Y. and Hochberg, Y. (1995), "Controlling the false discovery rate: a practical and powerful approach to multiple testing," Journal of the Royal Statistical Society, Series $B, 57,289-300$.

Besag, J., York, J., and Mollie, A. (1991), "A Bayesian image restoration, with two applications in spatial statistics (with discussion)," Annals of the Institute of Statistical Mathematics, 43, 1-59. 
Bithell, J. F. (1992), "Statistical methods for analysing point-source exposures," in Geographic and Environmental Epidemiology: Methods for Small Area Studies, eds. Elliot, P., Cuzick, D., English, D., and Stern, R., Oxford University Press: Oxford, pp. 1-35.

Bock, D., Andersson, E., and Frisén, M. (2008), "Statistical surveillance of epidemics: peak detection of influenza in Sweden," Biometrical Journal, 50, 71-85.

Bodnar, O. and Schmid, W. (2007), "Surveillance of the mean behavior of multivariate time series," Statistica Neerlandica, 61, 383-406.

Borror, C. M., Champ, C. W., and Rigdon, S. E. (1998), "Poisson EWMA control charts," Journal of Quality Technology, 30, 352-361.

Box, G. E. P. and Jenkins, G. M. (1970), Time Series Analysis: Forecasting and Control, Holden-Day: San Francisco.

Buckeridge, D. L., Burkom, H. S., Campell, M., Hogan, W. R., and Moore, A. (2005), "Algorithms for rapid outbreak detection: a research synthesis," Journal of Biomedical Informatics, 38, 99-113.

Burkom, H. S., Murphy, S., Coberly, J., and Hurt-Mullen, K. (2005), "Public health monitoring tools for multiple data streams," Morbidity and Mortality Weekly Report (Supplement), $54,55-62$.

Burkom, H. S., Murphy, S. P., and Shmueli, G. (2007), "Automated time series forecasting for biosurveillance," Statistics in Medicine, 26, 4202-4218.

Cappé, O., Moulines, E., and Rydén, T. (2005), Inference in Hidden Markov Models, Springer: New York.

Cardinal, M., Roy, R., and Lambert, J. (1999), "On the application of integer-valued time series models for the analysis of disease incidence," Statistics in Medicine, 18, 2025-2039.

Chatfield, C. and Yar, M. (1988), "Holt-Winters forecasting: some practical issues," The Statistician, 37, 129-140. 
Chen, R. (1978), "A surveillance system for congenital malformations," Journal of the American Statistical Association, 73, 323-327.

Chen, R., Connelly, R. R., and Mantel, N. (1993), "Analysing post-alarm data in a monitoring system in order to accept or reject the alert," Statistics in Medicine, 12, 1807-1812.

Chen, R., Iscovich, J., and Goldbourt, U. (1997), "Clustering of leukaemia cases in a city in Israel," Statistics in Medicine, 16, 1873-1887.

Choi, K. (1981), “An evaluation of influenza mortality surveillance, 1962-1979. 1: Time series forecasts of expected pneumonia and influenza deaths," American Journal of Epidemiology, 113, 215-226.

Clark, A. B. and Lawson, A. B. (2006), "Surveillance of individual level disease maps," Statistical Methods in Medical Research, 15, 353-362.

Conesa, D., López-Quílez, A., Martínez-Beneito, M. A., Miralles, M. T., and Verdejo, F. (2009), "FluDetWeb: an interactive web-based system for the early detection of the onset of influenza epidemics," BMC Medical Informatics and Decision Making, 9:36.

Conesa, D., Martínez-Beneito, M. A., Amorós, R., and López-Quílez, A. (2010), "Bayesian hierarchical Poisson model with a hidden Markov structure for the detection of influenza epidemic outbreaks," Working paper, Departament d'Estadística i Investigació Operativa, Universitat de València.

Cooper, D. L., Smith, G., Baker, M., Chinemana, F., Verlander, N. Q., Gerard, E., Hollyoak, V., and Griffiths, R. (2004), "National symptom surveillance using calls to a telephone health advice service - United Kingdom, December 2001-February 2003," Morbidity and Mortality Weekly Report (Supplement), 53, 179-183.

Costagliola, D., Flahault, A., Galinec, D., Garnerin, P., Menares, J., and Valleron, A.J. (1991), "A routine tool for detection and assessment of epidemics of influenza-like syndromes in France," American Journal of Public Health, 81, 97-99.

- (1994), "When is the epidemic warning cut-off point exceeded?" European Journal of Epidemiology, 10, 475-476. 
Cowling, B. J., Wong, I. O. L., Riley, S., and Leung, B. M. (2006), "Methods for monitoring influenza data," International Journal of Epidemiology, 35, 1314-1321.

Cox, D. R. (1955), "Some statistical methods related with series of events (with discussion)," Journal of the Royal Statistical Society Series B, 17, 129-157.

Crosier, R. B. (1988), "A new two-sided cumulative sum quality control scheme," Technometrics, 28, 187-194.

Dempster, A. P., Laird, N. M., and Rubin, D. B. (1977), "Maximum-likelihood from incomplete data via the EM algorithm," Journal of the Royal Statistical Society Series B, 39, $1-38$.

Diggle, P. J., Knorr-Held, L., Rowlingson, B., Su, T., Hawtin, P., and Bryant, T. (2004), "Spatio-temporal point processes: methods and applications," in Monitoring the Health of Populations: Statistical Principles $\&$ Methods for Public Health Surveillance, eds. Brookmeyer, R. and Stroup, D. F., Oxford University Press: Oxford, pp. 233-266.

Diggle, P. J., Rowlingson, B., and Su, T.-L. (2005), "Point process methodology for on-line spatio-temporal disease surveillance," Environmetrics, 16, 423-434.

Dominici, F., Sheppard, L., and Clyde, M. (2003), "Health effects of air pollution: a statistical review," International Statistical Review, 71, 243-276.

Dong, Y., Hedayat, A. S., and Sinha, B. K. (2008), "Surveillance stratgeies for detecting changepoint in incidence rate based on exponentially weighted moving average methods," Journal of the American Statistical Association, 103, 843-853.

Duczmal, L. and Assunção, R. M. (2004), "A simulated annealing strategy for the detection of arbitrarily shaped spatial clusters," Computational Statistics $\& 6$ Data Analysis, 45, 269 286.

Elbert, Y. and Burkom, H. S. (2009), "Development and evaluation of a data-adaptive alerting algorithm for univariate temporal biosurveillance data," Statistics in Medicine, $28,3226-3248$. 
Farrington, C. P. and Andrews, N. (2004), "Outbreak detection: Application to infectious disease surveillance," in Monitoring the Health of Populations: Statistical Principles $\&$ Methods for Public Health Surveillance, eds. Brookmeyer, R. and Stroup, D. F., Oxford University Press: Oxford, pp. 203-231.

Farrington, C. P., Andrews, N. J., Beal, A. D., and Catchpole, M. A. (1996), "A statistical algorithm for the early detection of outbreaks of infectious disease," Journal of the Royal Statistical Society Series A, 159, 547-562.

Fienberg, S. E. and Shmueli, G. (2005), "Statistical issues and challenges associated with rapid detection of bio-terrorist attacks," Statistics in Medicine, 24, 513-529.

Frisén, M. (2010), "Principles for multivariate surveillance," in Frontiers in Statistical Quality Cntrol 9, eds. Lenz, H.-J., Wilrich, P.-T., and W., S., Springer: Berlin, Heidelberg, pp. $133-144$.

Frisén, M. and Andersson, E. (2009), "Semiparametric surveillance of monotonic changes," Sequential Analysis, 28, 434-454.

Frisén, M., Andersson, E., and Pettersson, K. (2010a), "Semiparametric estimation of outbreak regression," Statistics, 44, 107-117.

Frisén, M., Andersson, E., and Schiöler, L. (2010b), "Sufficient reduction in multivariate surveillance," Communications in Statistics - Theory and Methods, to appear.

Frisén, M., Andersson, E., and Schiöler (2009), "Robust outbreak surveillance of epidemics in Sweden," Statistics in Medicine, 28, 476-493.

Frisén, M. and de Maré, J. (1991), "Optimal surveillance," Biometrika, 78, 271-280.

Gan, F. F. (1991), "Monitoring observations generated from a binomial distribution using modified exponentially weighted moving average control charts," Journal of Statistical Computation and Simulation, 37, 45-60.

— (1994), "Design of optimal exponential CUSUM control charts," Journal of Quality Technology, 26, 109-124. 
— (1998), "Designs of one- and two-sided exponential EWMA Charts," Journal of Quality Technology, 30, 44-69.

Glaz, J., Naus, J., and Wallenstein, W. (2001), Scan Statistics, Springer: New York.

Goldenberg, A., Shmueli, G., Caruana, R., and Fienberg, S. (2002), "Early statistical detection of anthrax outbreaks by tracking over-the-counter medication sales," Proceedings of the National Academy of Sciences of the United States, 99, 5237-5240.

Golosnoy, V., Ragulin, S., and Schmid, W. (2009), "Multivariate CUSUM chart: properties and enhancements," Advances in Statistical Analysis, 93, 263-279.

Griffin, B. A., Jain, A. K., Davies-Cole, J., Glymph, C., Lum, G., Washington, S. C., and Stoto, M. A. (2009), "Early detection of influenza outbreaks using the DC Department of Health's syndromic surveillance system," BMC Public Health, 9:483.

Han, S. W., Tsui, K.-L., Ariyajunya, B., and Kim, S. B. (2010), "A comparison of CUSUM, EWMA, and temporal scan statistics for detection of increases in Poisson rates," Quality and Reliability Engineering International, 26, 279-289.

Healy, M. J. R. (1983), "A simple method for monitoring routine statistics," The Statistician, $32,347-349$

Heisterkamp, S. H., Dekkers, A. L. M., and Heijne, J. C. M. (2006), "Automated detection of infectious disease outbreaks: hierarchical time series models," Statistics in Medicine, 25, 4179-4196.

Held, L., G., G., Frank, C., and Rue, H. (2006a), "Joint spatial analysis of gastrointestinal infectious diseases," Statistical Methods in Medical Research, 15, 465-480.

Held, L., Hofmann, M., Höhle, M., and Schmid, V. (2006b), "A two-component model for counts of infectious diseases," Biostatistics, 7, 422-437.

Held, L., Höhle, M., and Hofmann, M. (2005), "A statistical framework for the analysis of multivariate infectious disease surveillance counts," Statistical Modelling, 5, 187-199. 
Helfenstein, U. (1986), "Box-Jenkins modelling of some viral infectious diseases," Statistics in Medicine, 5, 37-47.

Höhle, M. (2007), "surveillance: An R package for the monitoring of infectious diseases," Computational Statistics, 22, 571-582.

Höhle, M. and Mazick, A. (2010), "Aberration detection in R illustrated by Danish mortality monitoring," in Biosurveillance: Methods and Case Studies, eds. Kass-Hout, T. and Zhang, X., CRC Press: London, to appear.

Höhle, M. and Paul, M. (2008), "Count data regression charts for the monitoring of surveillance time series," Computational Statistics \& Data Analysis, 52, 4357-4368.

Holt, C. C. (1957), "Forecasting trends and seasonals by exponentially weighted moving averages," ONR Memorandum 52/1957: Carnegie Institute of Technology.

Hotelling, H. (1947), "Multivariate quality control," in Techniques of Statistical Analysis, eds. Eisenhart, C., Hastay, M. W., and Wallis, W. A., New York: McGraw-Hill, pp. $111-184$.

Hulth, A., Andrews, N., Ethelberg, S., Dreesman, J., Faensen, D., van Pelt, W., and Schnitzler, J. (2010), "Practical usage of computer-supported outbreak detection in five European countries," Eurosurveillance, to appear.

Ismail, N. A., Pettitt, A. N., and Webster, R. A. (2003), "Online' monitoring and retrospective analysis of hospital outcomes based on a scan statistic," Statistics in Medicine, $22,2861-2876$.

Jackson, J. E. (1959), "Quality control methods for several related variables," Technometrics, $1,359-377$.

Jackson, M. J., Baer, A., Painter, I., and Duchin, J. (2007), "A simulation study comparing aberration detection algorithms for syndromic surveillance," BMC Medical Informatics and Decision Making, 7:6. 
Jacquez, G. M., Greilling, D. A., Durbeck, H., Estberg, L., Do, E., Long, A., and Rommel, B. (2002), "ClusterSeer User Guide 2: Software for Identifying Disease Clusters," TerraSeer Press: Ann Arbor, Michigan.

Jolliffe, I. T. (2002), Principal Component Analysis, Springer: New York, 2nd ed.

Joner Jr., M. D., Woodall, W. H., and Reynolds Jr, M. R. (2008), "Detecting a rate increase using a Bernoulli scan statistic," Statistics in Medicine, 27, 2555-2575.

Kafadar, K. and Stroup, D. (1992), "Analysis of aberrations in public health surveillance data: estimating variances on correlated samples," Statistics in Medicine, 11, 1551-1568.

Kenett, R. S. and Pollak, M. (1996), "Data-analytic aspects of the Shiryaev-Roberts control chart: surveillance of a non-homogeneous Poisson process," Journal of Applied Statistics, $23,125-137$.

Kleinman, K. P., Abrams, A., Kulldorff, M., and Platt, R. (2005), "A model-adjusted spacetime scan statistic with an application to syndromic surveillance," Epidemiology and Infection, 133, 409-419.

Kleinman, K. P., Lazarus, R., and Platt, R. (2004), "A generalized linear mixed models approach for detecting incident clusters of disease in small areas, with an application to biological terrorism," American Journal of Epidemiology, 159, 217-224.

Knorr-Held, L. (2000), "Bayesian modelling of inseparable space-time variation in disease risk," Statistics in Medicine, 19, 2555-2567.

Knox, E. G. (1964), "The detection of space-time interactions," Applied Statistics, 13, 25-29.

$\mathrm{Ku}, \mathrm{W}$., Storer, R., and Georgakis, C. (1995), "Disturbance detection and isolation by dynamic principal component analysis," Chemometrics and Intelligent Laboratory Systems, 30, 179-196.

Kulldorff, M. (1997), "A spatial scan statistic," Communications in Statistics: Theory and Methods, 26, 1481-1496. 
— (2001), "Prospective time periodic geographical disease surveillance using a scan statistic," Journal of the Royal Statistical Society Series A, 164, 61-72.

- (2010), "SaTScan version 8.2.1: software for the spatial, temporal, and space-time scan statistics," http://www.satscan.org.

Kulldorff, M., Athos, W., Feuer, E., Miller, B., and Key, C. (1998), "Evaluating cluster alarms: a space-time scan statistic and brain cancer in Los Alamos," American Journal of Public Health, 188, 1377-1380.

Kulldorff, M., Heffernan, R., Hartman, J., Assunção, R., and Mostashari, F. (2005), "A space-time permutation scan statistic for disease outbreak detection," PLoS Medicine, 2, $216-224$.

Kulldorff, M., Huang, L., Pickle, L., and Duczmal, L. (2006), "An elliptic spatial scan statistic," Statistics in Medicine, 25, 3929-3943.

Kulldorff, M., Mostashari, F., Duczmal, L., Yih, W. K., Kleinman, K., and Platt, R. (2007), "Multivariate scan statistics for disease surveillance," Statistics in Medicine, 26, 18241833.

Lawson, A. B. (2005), "Spatial and spatio-temporal disease analysis," in Spatial and Syndromic Surveillance for Public Health, eds. Lawson, A. B. and Kleinman, K., John Wiley \& Sons: Chichester, pp. 55-76.

Lawson, A. B., Clark, A., and Rodeiro, C. L. V. (2003), "Developments in general and syndromic surveillance for small area health data," Journal of Applied Statistics, 31, 951966.

Le Strat, Y. and Carrat, F. (1999), "Monitoring epidemiologic surveillance data using hidden Markov models," Statistics in Medicine, 18, 3463-3478.

Lowry, C. A., Woodall, W. H., Champ, C. W., and Rigdon, S. E. (1992), "A multivariate exponentially weighted moving average control chart," Technometrics, 34, 46-53. 
Lu, H.-M., Zeng, D., and Chen, H. (2010), "Prospective infectious disease outbreak detection using Markov switching models," IEEE Transactions on Knowledge $\&$ Data Engineering, $22,565-577$.

Lucas, J. M. (1985), "Counted data CUSUM's," Technometrics, 27, 129-144.

Madigan, D. (2005), "Bayesian data mining for health surveillance," in Spatial and Syndromic Surveillance for Public Health, eds. Lawson, A. B. and Kleinman, K. P., John Wiley \& Sons: Chicester, pp. 203-221.

Mann, A. E. (2009), "Estimating the impact of influenza vaccinations and antigenic drift on influenza-related morbidity and mortality in England and Wales using hidden Markov models," PhD Thesis, University of London.

Marshall, C., Best, N., Bottle, A., and Aylin, P. (2004), "Statistical issues in the prospective monitoring of health outcomes across multiple units," Journal of the Royal Statistical Society Series A, 167, 541-559.

Marshall, J. B., Spitzner, D. J., and Woodall, W. H. (2007), "Use of the local Knox statistic for the prospective monitoring of disease occurrences in space and time," Statistics in Medicine, 26, 1579-1593.

Martínez-Beneito, M. A., Conesa, D., López-Quílez, A., and López-Maside, A. (2008), "Bayesian Markov switching models for the early detection of influenza epidemics," Statistics in Medicine, 27, 4455-4468.

McCabe, G. J., Greenhalgh, D., Gettingby, G., Holmes, E., and Cowden, J. (2003), "Prediction of infectious diseases: an exception reporting system," Journal of Medical Informatics and Technologies, 5, 67-74.

Miller, B., Kassenborg, H., Dunsmuir, W., Griffith, J., Hadidi, M., Nordin, J., and Danila, R. (2003), "Syndromic surveillance for influenzalike illness in ambulatory care network," Emerging Infectious Diseases, 10, 1806-1811.

Montgomery, D. C. (2009), Introduction to Statistical Process Control, John Wiley \& Sons: Hoboken, New Jersey, 6th ed. 
Mostashari, F., Kulldorff, M., Hartman, J. J., Miller, J. R., and Kulasekera, V. (2003), "Dead bird clusters as an early warning system for West Nile virus activity," Emerging Infectious Diseases, 9, 641-646.

Munford, A. G. (1980), "A control chart based on cumulative scores," Applied Statistics, 26, $252-258$.

Naus, J. and Wallenstein, S. (2006), "Temporal surveillance using scan statistics," Statistics in Medicine, 25, 311-324.

Ngai, H.-M. and Zhang, J. (2001), "Multivariate cumulative sum control charts based on projection pursuit," Statistica Sinica, 11, 747-766.

Ngo, L., Tager, I. B., and Hadley, D. (1996), "Application of exponential smoothing for nosocomial infection surveillance," American Journal of Epidemiology, 143, 637-647.

Nobre, F. F. and Stroup, D. F. (1994), "A monitoring system to detect changes in public health surveillance data," Journal of Epidemiology, 23, 408-418.

Oakland, J. S. (2008), Statistical Process Control, Butterworth-Heinemann: Oxford, 6th ed.

Page, P. E. (1954), "Continuous inspection schemes," Biometrika, 41, 100-115.

Parker, R. A. (1989), "Analysis of surveillance data with Poisson regression: a case study," Statistics in Medicine, 8, 285-294.

Paul, M., Held, L., and Toschke, A. M. (2008), "Multivariate modelling of infectious disease surveillance data," Statistics in Medicine, 27, 6250-6267.

Pelat, C., Boëlle, P.-Y., Cowling, B. J., Carrat, F., Flahault, A., Ansart, S., and Valleron, A.J. (2007), "Online detection and quantification of epidemics," BMC Medical Informatics and Decision Making, 7:29.

Pignatiello Jr., J. J. and Runger, G. C. (1990), "Comparison of multivariate CUSUM charts," Journal of Quality Technology, 22, 173-186.

Qiu, P. H. and Hawkins, D. (2001), "A rank-based multivariate CUSUM procedure," Technometrics, 43, 120-132. 
— (2003), "A nonparametric multivariate cumulative sum procedure for detecting shifts in all directions," The Statistician, 52, 151-164.

Rath, T. M., Carreras, M., and Sebastiani, P. (2003), "Automated detection of influenza epidemics with hidden Markov models," in Advances in Intelligent Data Analysis V, LNCS 2810, eds. Berthold, M. R., Lenz, H.-J., Bradley, E., Kruse, R., and Borgelt, C., Springer: Berlin, pp. 521-532.

Raubertas, R. F. (1989), "An analysis of disease surveillance data that uses the geographical locations of the reporting units," Statistics in Medicine, 8, 267-271.

Reis, B. Y. and Mandl, K. D. (2003), "Time series modeling for syndromic surveillance," BMC Medical Informatics and Decision Making, 3, No. 2.

Reynolds Jr., M. R. and Stoumbos, Z. G. (2000), “A general approach to modeling CUSUM charts for a proportion," IIE Transactions, 32, 515-535.

Roberts, S. W. (1959), "Control chart tests based on geometric moving averages," Technometrics, 1, 239-250.

- (1966), "A comparison of some control chart procedures," Technometrics, 8, 411-430.

Robertson, C. (2006), "Protecting the leaders: syndromic surveillance for the G8 summit in Scotland," Significance, 3, 69-72.

Rogerson, P. A. (1997), "Surveillance systems for monitoring the development of spatial patterns," Statistics in Medicine, 26, 2081-2093.

- (2001), "Monitoring point patterns for the development of spatial patterns," Journal of the Royal Statistical Society Series A, 164, 87-96.

Rogerson, P. A. and Yamada, I. (2004a), "Approaches to syndromic surveillance when data consist of small regional counts," Morbidity and Mortality Weekly Report (Supplement), $53,79-85$.

- (2004b), "Monitoring change in spatial patterns of disease: comparing univariate and multivariate cumulative sum approaches," Statistics in Medicine, 23, 2195-2214. 
Rossi, G., Lampugnani, L., and Marchi, M. (1999), "An approximate CUSUM procedure for surveillance of health events," Statistics in Medicine, 18, 2111-2122.

Schiöler, L. and Frisén, M. (2010), "Multivariate outbreak detection," Manuscript submitted for publication.

Sebastiani, P., Mandl, K. D., Szolovits, P., Kohane, I. S., and Ramoni, M. F. (2006), “A Bayesian dynamic model for influenza surveillance," Statistics in Medicine, 25, 1803-1816.

Sego, L. H., Woodall, W. H., and Reynolds Jr, M. R. (2008), “A comparison of surveillance methods for small incidence rates," Statistics in Medicine, 27, 1225-1247.

Serfling, R. (1963), "Methods for current statistical analysis of excess pneumonia-influenza deaths," Public Health Reports, 78, 494-506.

Shewhart, W. A. (1931), Economic Control of Quality of Manufactured Product, Van Nostrand Reinhold: Princeton.

Shiryaev, A. N. (1963), "On the detection of disorder in a manufactoring process," Theory of Probability and its Applications, 8, 247-265.

Shmueli, G. (2005), "Wavelet-based monitoring for modern biosurveillance," Working paper, RHS-06-002: Robert H. Smith School of Business, University of Maryland. http://ssrn.com/abstract $=902878$.

Shmueli, G. and Burkom, H. (2010), "Statistical challenges facing early outbreak detection in biosurveillance," Technometrics, 52, 39-51.

Shore, D. L. and Quade, D. (1989), "A surveillance system based on a short memory scheme," Statistics in Medicine, 8, 311-322.

Sitter, R. R., Hanrahan, L. P., Anderson, H. A., and DeMets, D. (1991), "The authors' reply," American Journal of Epidemiology, 134, 917-919.

Sitter, R. R., Hanrahan, L. P., DeMets, D., and Anderson, H. A. (1990), "A monitoring system to detect increased rates of cancer incidence," American Journal of Epidemiology, $132,123-130$. 
Sonesson, C. (2003), "Evaluations of some exponentially weighted moving average methods," Journal of Applied Statistics, 30, 1115-1133.

- (2007), "A CUSUM framework for detection of space-time disease clusters using scan statistics," Statistics in Medicine, 26, 4770-4789.

Sonesson, C. and Bock, D. (2003), "A review and discussion of prospective statistical surveillance in public health," Journal of the Royal Statistical Society Series A, 166, 5-21.

Sonesson, C. and Frisén, M. (2005), "Multivariate surveillance," in Spatial Surveillance for Public Health, eds. Lawson, A. B. and Kleinman, K., John Wiley \& Sons: Chicester, pp. $169-186$.

Sparks, R. (2010), "Enhancing road safety through early detection of outbreaks in the frequency of motor vehicle crashes," Safety Science, 48, 135-144.

Stern, L. and Lightfoot, D. (1999), "Automated outbreak detection: A quantitative retrospective analysis," Epidemiology and Infection, 122, 103-110.

Steutel, F. W. and van Harn, K. (1979), "Discrete analogues of self-decomposability and stability," Annals of Probability, 7, 893-899.

Stroup, D., Wharton, M., Kafadar, K., and Dean, A. (1993), "Evaluation of a method for detecting aberrations in public health surveillance data," American Journal of Epidemiology, $137,373-380$.

Stroup, D. F., Thacker, S. B., and Herndon, J. L. (1988), "Application of multiple time series analysis to the estimation of pneumonia and influenza mortality by age 1962-1983," Statistics in Medicine, 7, 1045-1059.

Stroup, D. F., Williamson, G. D., Herndon, J. L., and Karon, J. M. (1989), "Detection of aberrations in the occurence of notifiable diseases surveillance data," Statistics in Medicine, 8, 323-329.

Tango, T. Takahashi, K. (2005), "A flexibly shaped spatial scan statistic for detecting clusters," International Journal of Health Geographics, 4:11. 
Tango, T. (1995), "A class of tests for detecting general and focussed clustering of rare diseases," Statistics in Medicine, 14, 2323-2334.

Tillett, H. E. and Spencer, I.-L. (1982), "Influenza surveillance in England and Wales using routine statistics," Journal of Hygiene, Cambridge, 88, 83-94.

Vidal Rodeiro, C. L. and Lawson, A. B. (2006), "Monitoring changes in spatio-temporal maps of disease," Biometrical Journal, 3, 463-480.

Wagner, A. (2010), "Extending Scottish Exception Reporting Systems Spatially and Temporally," Ph.D. thesis, University of Strathclyde.

Wallenstein, S. (1980), "A test for detection of clustering in time," American Journal of Epidemiology, 111, 367-372.

Wallenstein, S., Gould, M., and Kleinman, M. (1989), "Use of the scan statistic to detect time-space clustering," American Journal of Epidemiology, 130, 1057-1064.

Wallenstein, S. and Naus, J. (2004), "Scan statistics for temporal surveillance for biologic terrorism," Morbidity and Mortality Weekly Report (Supplement), 53, 74-78.

Watier, L., Richardson, S., and Hubert, B. (1991), "A time series construction of an alert threshold with application to S. bovismorbificans in France," Statistics in Medicine, 10, 1493-1509.

Watkins, R. E., Eagleson, S., Veenendaal, B., Wright, G., and Plant, A. J. (2009), "Disease surveillance using a hidden Markov model," BMC Medical Informatics and Decision Making, 9:39.

Weiß, C. H. (2009), Categorical Time Series Analysis and Applications in Statistical Quality Control, dissertation.de Verlag: Berlin.

West, M. and Harrison, J. (1997), Bayesian Forecasting and Dynamic Models, Springer: New York, 2nd ed.

Widdowson, M.-A., Bosman, A., van Straten, E., Tinga, M., Chaves, S., van Eerden, L., and van Pelt, W. (2003), "Automated, laboratory-based system using the internet for disease outbreak detection," Emerging Infectious Diseases, 9, 1046-1052. 
Wieland, S. C., Brownstein, J. S., Berger, B., and Mandl, K. D. (2007), "Automated realtime constant specificity surveillance for disease outbreaks," BMC Medical Informatics and Decision Making, 7:15.

Williamson, G. and Weatherby Hudson, G. (1999), "A monitoring system for detecting aberrations in public health surveillance reports," Statistics in Medicine, 18, 3283-3298.

Winters, P. R. (1960), "Forecasting sales by exponentially weighted moving averages," Management Science, 6, 324-342.

Wolter, C. (1987), "Monitoring intervals between rare events: a cumulative score procedure compared with Rina Chen's sets technique," Methods of Information in Medicine, 26, $215-219$.

Woodall, W. H. (2006), "Use of control charts in health care and public health surveillance (with discussion)," Journal of Quality Technology, 38, 88-103.

Woodall, W. H., Marshall, J. B., Joner Jr., M. D., Fraker, S. E., and Abdel-Salam, A.S. G. (2008), "On the use and evaluation of prospective scan methods for health-related surveillance," Journal of the Royal Statistical Society Series A, 171, 223-237.

Yamada, I., Rogerson, P., and Lee, G. (2009), "GeoSurveillance: a GIS-based system for the detection and monitoring of spatial clusters," Journal of Geographical Systems, 11, $155-173$.

Zhang, J., Tsui, F.-C., Wagner, M. M., and Hogan, W. (2003), "Detection of outbreaks from time series data using wavelet transform," AMIA Annual Symposium, Omnipress: Madison, Wisconsin, 748-552.

Zhang, T. and Lin, G. (2009), "Spatial scan statistics in loglinear models," Computational Statistics $\&$ Data Analysis, 53, 2851-2858.

Zhou, H. and Lawson, A. B. (2008), "EWMA smoothing and Bayesian spatial modeling for health surveillance," Statistics in Medicine, 27, 5907-5928. 